\title{
Response Time Driven Multimedia Data Objects Allocation for Browsing Documents in Distributed Environments
}

\author{
Siu-Kai So, Ishfaq Ahmad, Member, IEEE, and Kamalakar Karlapalem, Member, IEEE
}

\begin{abstract}
Distributed information processing, in many world wide web applications, requires access, transfer, and synchronization of large multimedia data objects (MDOs) (such as, audio, video, and images) across the communication network. Moreover, the end users have started expecting very fast response times and high quality of service. Since the transfer of large MDOs across the communication network contributes to the response time observed by the end users, the problem of allocating these MDOs so as to minimize the response time becomes very challenging. This problem becomes more complex in the context of hypermedia documents (web pages), wherein the MDOs need to be synchronized during presentation to the end users. Note that the basic problem of data allocation in distributed database environments is NP-complete. Therefore, there is a need to pursue and evaluate solutions based on heuristics which generate near-optimal MDO allocation. In this paper, we address this problem by: 1) conceptualizing this problem by using a navigational model to represent hypermedia documents and their access behavior from end users, and by capturing the synchronization requirements on MDOs, 2) formulating the problem by developing a base case cost model for response time, and generalizing it to incorporate user interaction and buffer memory constraints, 3) designing two algorithms to find near-optimal solutions for allocating MDOs of the hypermedia documents while adhering to the synchronization requirements, and 4) evaluating the trade-off between the time complexity to get the solution and the quality of solution by comparing the solutions generated by the algorithms with the optimal solutions generated through an exhaustive search.
\end{abstract}

Index Terms-Data allocation, response time, multimedia data objects, hypermedia documents, distributed hypermedia document systems, navigational model.

\section{INTRODUCTION}

$\mathrm{D}$ ISTRIBUTED information processing has become the norm in recent years. Most of the Internet driven web based information access requires distributed processing. In many applications, this processing typically requires, access, transfer and synchronization of multimedia data objects (MDOs) (such as, audio, video, and images) [1], [5]. The quality of services provided in presenting these MDOs to end users has become an issue of paramount importance. End users have started expecting strict adherence to synchronization and response time constraints. Any application or system which cannot respond quickly and in a timely manner for presenting MDOs to end users is at a clear disadvantage. In order to manage and present large number of hypermedia documents and their MDOs distributed hypermedia database systems have been proposed [19]. In fact, a set of web servers can be treated as a distributed hypermedia database system. Therefore, the solutions and the approaches developed in this paper can also be applied in designing efficient web servers in intranet environments (wherein the organization has a complete control in placing the web pages at different internal web servers).

\footnotetext{
- The authors are with the Department of Computer Science, Hong Kong University of Science and Technology, Clear Water Bay, Kowloon, Hong Kong. E-mail: iahmad@cs.ust.hk.
}

Manuscript received 15 November 1997; revised 7 July 1998.

For information on obtaining reprints of this article, please send e-mail to: tkde@computer.org, and reference IEEECS Log Number 107202.
Distributed database systems have introduced a number of problems, such as the data fragmentation and data allocation problems, that do not exist in centralized database systems [12]. A good data allocation scheme is always highly desirable, even in single-media distributed database systems, since it can significantly reduce the response time of database queries. Due to the large variations in the sizes of MDOs such a data allocation scheme is even more urgently needed for distributed hypermedia database systems. These systems also cater to high performance applications wherein a set of end users can access multiple hypermedia documents in any order and expect good response time and quality of service. Since the hypermedia documents may not be located at the end users' sites, they need to be transferred across the communication network incurring delays (increasing response time) in presenting the MDOs of the hypermedia documents. Since end users at different sites may access the same hypermedia documents, the problem of hypermedia data allocation gets further complicated. Hence the allocation of the hypermedia documents and their MDOs govern the response time for the end users. Further, since the MDOs in a hypermedia document need to be synchronized, the allocation should also adhere to these synchronization constraints.

We model a hypermedia document as a directed graph with each node representing a hypermedia document with its MDOs, and each out going directed edge as a hyperlink to another hypermedia document. Because of the vagaries of the communication network, the MDOs are presented to 


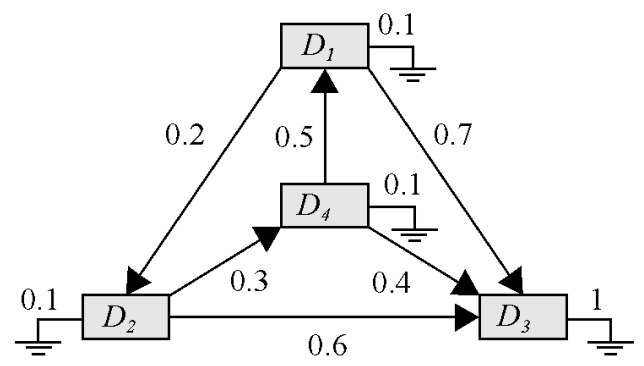

Nav $=\begin{gathered}D_{1} \\ D_{1} \\ D_{2} \\ D_{3} \\ D_{4}\end{gathered}\left[\begin{array}{cccc}0 & 0.2 & 0.7 & 0 \\ 0 & 0 & 0.6 & 0.3 \\ 0 & 0 & 0 & 0 \\ 0.5 & 0 & 0.4 & 0\end{array}\right]$

Fig. 1. Probability model of navigational links between hypermedia documents.

the end users only after they are buffered at the end-users' site. Therefore, the synchronization requirements may impose additional delays for presenting the MDOs to the end users [13], [26]. We also take into consideration end-user behavior in accessing the hypermedia documents from various sites, and the frequencies with which they access these hypermedia documents. We then develop a cost model which takes into consideration the synchronization constraints for calculating the response time for the end users for a given allocation scheme. We present two algorithms for finding the near-optimal data allocation of hypermedia documents. Subsequently, we illustrate our model and approach by a real-life example and evaluate the goodness of the proposed algorithms in terms solution quality (by comparing with the optimal solution) and time complexity in achieving this solution.

We design and evaluate data allocation algorithms so as to optimize the response time for a set of end users while adhering to the synchronization requirements of the MDOs presentation in distributed hypermedia database systems. In Section 2, we introduce different modeling specifications of multimedia documents. In the same section we propose a graphical notion to represent navigation in the hypermedia systems. In Section 3, we develop a cost model for the data allocation problem of distributed hypermedia systems. This cost model is used to evaluate an example hypermedia database system. In Section 4, we describe the proposed algorithms based on Hill-Climbing and probabilistic neighborhood search approaches. In Section 5, we include the experimental results. In Section 6, we provide an overview of existing related work as well as other issues related to our problem. Section 7 presents conclusions and possible extensions to this work.

\section{Modeling Hypermedia Documents}

A hypermedia document is a directed graph $D G(H, E)$ where $H=\left\{D_{1}, D_{2}, \ldots, D_{n}\right\}$ is the set of vertices, each $D_{p}$ representing a hypermedia document, and each directed edge from $D_{p}$ to $D_{p}$, is a link denoting access of document $D_{p}$, from document $D_{p}$. Therefore, a user can start browsing the documents from (say) document $D_{p}$ and then proceed to access document $D_{p}$, etc. Further, each hypermedia document has a set of MDOs which need to be presented to the end users accessing this document. Since the end users can access the hypermedia documents in any order and browse through them, we have a label attached to each directed edge from $D_{p}$ to $D_{p}$, giving the probability of end users accessing document $D_{p}$, from document $D_{p}$. These probabilities can be generated by gathering statistics (about document access, and browsing through logs of users browsing activity) about end-user behavior over a period of time. Further, since a user may end browsing after accessing any hypermedia document, the probabilities of outgoing edges from a vertex do not add up to 1.0, and the difference is the probability of ending the browsing at document $D_{p}$, and is shown by an edge connecting to the ground (see Fig. 1). An $n \times n$ matrix Nav is used to capture this information.

EXAMPLE 1. Suppose we have four hypermedia documents $D_{1}-D_{4}$, Fig. 1 shows the links between these documents and the probabilities of access from one document to another. Further, we also show the probability of ending the browsing session at each hypermedia document. For instance, there is a probability of 0.1 that browsing ends after accessing document $D_{1}$. The corresponding Nav matrix is shown on the right-hand side (of Fig. 1).

From the above navigational model, we can calculate the cumulative long run probabilities of accessing a hypermedia document $D_{p}$, from document $D_{p}$. This is done by considering all possible paths from document $D_{p}$ to $D_{p^{\prime}}$, and calculating the probability of accessing $D_{p}$, from document $D_{p}$ for each path, and taking the maximum of all these probabilities. Note that we assume each document access and browsing from one document to another to be independent events. Therefore, for a path with $t$ edges from document $D_{p}$ to document $D_{p^{\prime}}$, the probability of this path is the product of $t$ probabilities for the edges. Since there can be potentially infinitely long paths, we limit the length of the path by limiting the value of the cumulative probability given by the path to be greater than a parameter value $(b p l)$. Let $R$ be the $n \times n$ matrix, with each element $r_{p p}$, giving the cumulative long run probability of accessing document $D_{p}$, from document $D_{p}$.

EXAMPLE 1 (continued). From the navigational model, we can construct a tree for each document representing the possible navigation path for each session starting from that document. These are given in Fig. 2. We set the $b p l$ value to be 0.01 . Notice that we do not need to further expand a node if the document represented by that node is the same as that of the root. (This happens in the first tree in Fig. 2). Therefore, if we start navigating the hypermedia system from document $D_{1}$, we have probability 0.2 that we browse 

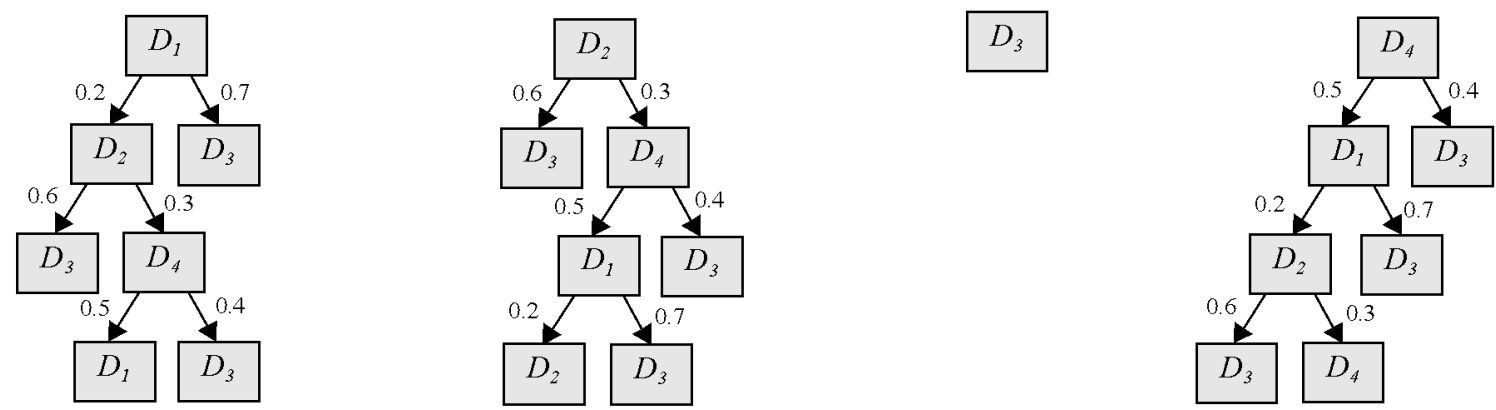

Fig. 2. Navigation paths starting from each hypermedia document ( $b p /$ is set as 0.01 ).

document $D_{2}$. For document $D_{3}$, if we follow the right path from $D_{1}$, the probability is equal to 0.7 . But if we follow the path $D_{1} \rightarrow D_{2} \rightarrow D_{3}$, the probability is equal to $0.2 \times 0.6=0.12$. In this case, we use the greater probability to represent the long run probability of browsing $D_{3}$ from $D_{1}$ as 0.7 .

Similarly other cumulative probability values are calculated. Therefore, the matrix $R$ is

$$
\begin{aligned}
& \begin{array}{llll}
D_{1} & D_{2} & D_{3} & D_{4}
\end{array} \\
& R=\begin{array}{c}
D_{1} \\
D_{2} \\
D_{3} \\
D_{4}
\end{array}\left[\begin{array}{cccc}
1 & 0.20 & 0.70 & 0.06 \\
0.15 & 1 & 0.60 & 0.30 \\
0 & 0 & 1 & 0 \\
0.50 & 0.10 & 0.40 & 1
\end{array}\right] .
\end{aligned}
$$

We use the Object Composition Petri Nets (OCPNs) [13] for modeling the synchronization constraints among the MDOs in a hypermedia document. Petri nets are powerful tools for representing objects that must be synchronized. In addition, they have the advantage of generating database schema as well as extracting spatio-temporal and content semantics [5]. In contrast to HyTime [6], Petri nets use a graphical notation for representing synchronization constraints. The hyperlinks are associated with the transitions [21] of the Petri net. With this mechanism, we can easily maintain a hyperlink by storing the address of the destination document in the database [10] and we will no longer be concerned about invalid links when the document addresses are changed. Further, $O C P N$ simplifies the Petri nets by restricting the number of outgoing edges from each transition to two and enhances them by introducing the duration and the addressing scheme for each place. This enhancement makes OCPN suitable for modeling synchronization constrains among MDOs of hypermedia documents. We can transverse a transition (called as firing) if all places pointing to this transition have a token and are in an unlocked state. When the transition fires, the places that the transition is pointing to will become active (a token is added to these places) and locked. Places will become unlocked when their durations have expired. All OCPN models can be mapped to a corresponding HyTime model [3]. In Fig. 3, the following synchronization constraint is represented: MDO $A$ has to be shown exactly 10 time units after the start of the presentation of the hypermedia document, and after another 30 time units MDO $B$ must be shown.

In [13] the multimedia specification associates time with a place. However, in traditional timed-Petri net, time is associated with a transition [15]. The reason for associating time with a place is for compactness. By using the traditional method, we can model user interaction [21] during hypermedia document presentation. In Fig. 4 the presentation of MDO $D$, represented as a box, will continue as long as both of the two places pointing to it have tokens. The user can interrupt the presentation by pressing the button associated with the immediate transition, represented in Fig. 4 by a bold vertical line. This user interaction will fire the upper transition, removing the token in the middle place on the left.

EXAMPLE 1 (continued). The OCPN synchronization specifications of hypermedia documents $D_{1}$ to $D_{4}$ are shown in Fig. 5.

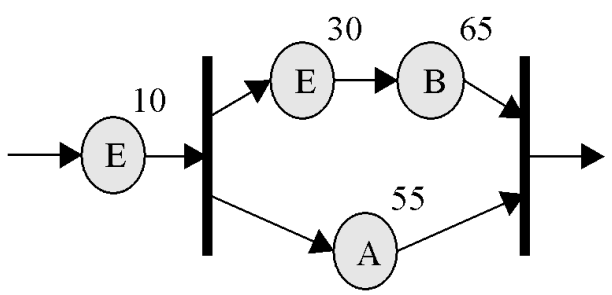

Fig. 3. The OCPN model of synchronizing MDOs A and $\mathrm{B}$, where $\mathrm{E}$ represents some delay event.

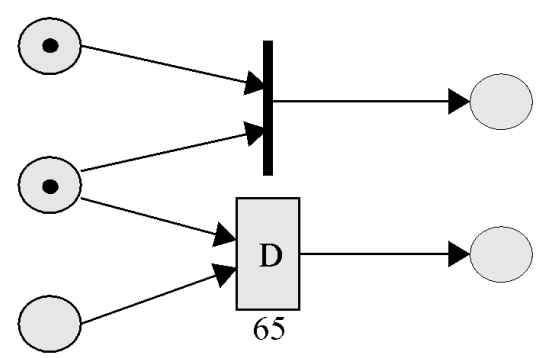

Fig. 4. Traditional timed-Petri net modeling of user interaction of presentation. 

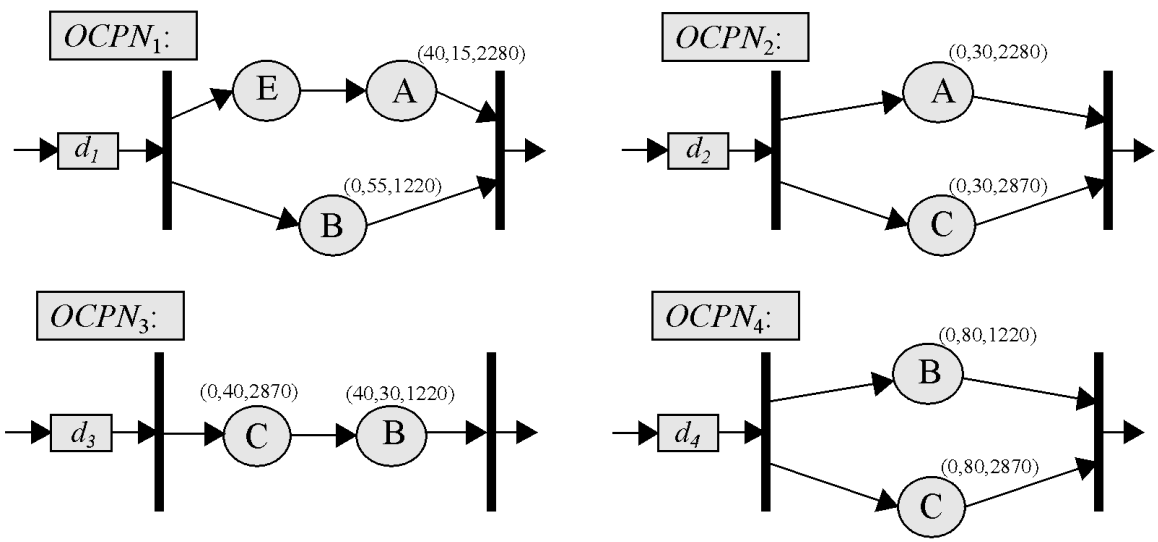

Fig. 5. The OCPN specification of each hypermedia document; the tuple is [start time, duration, media size in kilobytes].

\section{Cost Model for Data Allocation Scheme}

In order to reduce response time for the end users browsing activities, we need to develop a cost model for calculating the total response time observed. This response time depends on the location of the MDOs and the location of the end user. Further, it depends on the synchronization constraints among the MDOs of the hypermedia document browsed. The hypermedia document navigational model presented in Section 2 is used to estimate the number of accesses (times browsed) to each MDO from each site. This gives us the information regarding the affinity between the MDOs and the sites of the distributed environment. Typically, one would assign an MDO to a site which accesses it the most. But this may incur large delay for other sites that also need to access this MDO. Further, synchronization constraints may impose additional delays in transferring the MDO to the end-user site. This is done when two streams of MDOs need to simultaneously finish their presentation, and one of them is for shorter duration than the other. Since we buffer the MDOs at the user sites before the start of the presentation, the MDO allocation problem needs to minimize the additional delay incurred because of the synchronization constraints. We also take into consideration limited buffer space constraint at end user's site and user interaction during MDO presentation.

Table 1 lists a number of notations used throughout this paper.

\subsection{Total Response Time Cost Function}

Suppose there are $m$ sites in the distributed hypermedia database system. Let $S_{i}$ be the name of site $i$ where $1 \leq i \leq m$. These $m$ sites are connected by a communication network. A communication link between two sites $S_{i}$ and $S_{i}$, will have a positive integer $c_{i i}$, associated with it giving the transmission speed from site $i$ to site $i^{\prime}$. Note that these values depend on the routing scheme of the network. If fixed routing is used, we can get the exact values. However, if dynamic routing is used, we can only obtain the expected values statistically. Let there be $j$ hypermedia documents, called $\left\{D_{1}, D_{2}, \ldots, D_{j}\right\}$ accessing $k$ MDOs, named $\left\{O_{1}, O_{2}, \ldots, O_{k}\right\}$.
EXAMPLE 1 (continued). Assume that the hypermedia database system for storing the MDOs is distributed in a network with three sites. The transmission speeds between the three sites can be represented as an $m \times m$ matrix $C$, with entry $c_{i i}$, representing the transmission speed from $S_{i}$ to $S_{i}$.

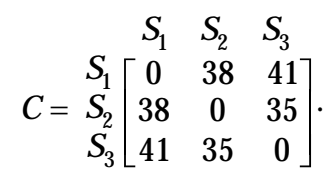

As explained above, from the navigation model, we can construct $j$ trees representing the navigation path of the session starting from each document. Since the height of these trees will typically be infinite, we must limit the level we will use for our cost model. We limit the height of the trees such that the cumulative probability of each path is greater than a threshold value $b p l$, say 0.001 . These trees will give us cumulative long run probability $r_{j j}$ of retrieving the document $D_{j}$, if we start navigating from the document $D_{j}$.

For each site, we use an irreducible continuous-timed Markov process [23] to model the user behavior across browsing sessions as a stationary regular transition probability matrix, $P^{i}, 1 \leq i \leq m$. These processes will converge in the long run and from these long run behaviors, we can estimate the probability of using each document as starting point for each browser session initiated in each site. These Markov chains will have $n+1$ states representing the probabilities of using each of the documents as the starting point for browsing session ( $n$, states), and probability of not browsing any of the documents $((n+1)$ th state, shown by row/column $E$ in matrices $P^{i}$ below). After analyzing the long run behavior of the Markov chain at each site, we will have the probabilities of using each document as initial browsing document and of not browsing at each site. As there is no delay when the user does not browse, we can eliminate the probability of not browsing. If we normalize the probabilities derived from long run behavior of Markov chain at each site and multiply them by a constant vector $F$ (number of accesses to documents at each site), we get the expected frequencies of initiating browsing at each 
TABLE 1

SYMBOLS AND THEIR MEANINGS

\begin{tabular}{|c|c|}
\hline Symbol & Meaning \\
\hline$\overline{S_{i}}$ & The ith site \\
\hline$D_{j}$ & The $j$ th hypermedia document \\
\hline$O_{k}$ & The $k$ th $M D O$ \\
\hline$m$ & The number of sites in the network \\
\hline$n$ & The number of hypermedia documents in the database system \\
\hline$k$ & The number of $M D O$ s in the database system \\
\hline $\bar{B}$ & The navigation initial document frequencies matrix \\
\hline$\overline{b_{i j}}$ & The frequency of using the $j$ th document as initial point at the ith site \\
\hline $\mathrm{C}$ & The transmission speed matrix of the network \\
\hline$c_{i i^{\prime}}$ & The transmission speed from site $i$ to site $i^{\prime}$ \\
\hline$P^{i}$ & The user navigation probability matrix of site $i$ \\
\hline$p_{j j^{\prime}}^{i}$ & $\begin{array}{l}\text { The probability matrix modeling the user behavior at site } i \text { between browsing } \\
\text { sessions. This value gives the probability of using document } j^{\prime} \text { as initial browsing } \\
\text { document in the next session if the initial browsing document is } j \text { in the current } \\
\text { navigation session }\end{array}$ \\
\hline A & The access frequencies matrix \\
\hline$a_{i j}$ & The access frequency of document $j$ from site $i$ \\
\hline$l$ & The allocation limit vector of the sites \\
\hline$\overline{l_{i}}$ & The allocation limit of site $i$ \\
\hline$R$ & The hypermedia document dependency matrix \\
\hline$r_{j j^{\prime}}$ & The probability of retrieving document $j^{\prime}$ if browsing initial document is $j$ \\
\hline$O C P N_{j}$ & The OCPN specification of document $j$ \\
\hline$U$ & The usage matrix \\
\hline$u_{j k}$ & The boolean value of whether document $j$ uses $M D O k$ \\
\hline$d u r_{j k}$ & The presentation duration of $M D O k$ in document $j$ \\
\hline start $_{j k}$ & The presentation starting time of $M D O k$ in document $j$ \\
\hline $\operatorname{size}_{k}$ & The size of the $k$ th $M D O$ \\
\hline$b p l$ & The browsing probability limit \\
\hline$e t_{j}$ & The expected number of times document $j$ will be retrieved \\
\hline$D$ & The delay matrix \\
\hline$d_{i j}$ & The delay of presentation starting time of document $j$ at site $i$ \\
\hline$F$ & The access frequency vector \\
\hline Nav & The navigation probability between documents within each browsing session \\
\hline$t$ & The total delay \\
\hline
\end{tabular}

document from each site in unit time. The resultant information is represented by an $m \times n$ matrix $B$.

We multiply this matrix to the $n \times n$ matrix $R$ obtained from the hypermedia document trees to generate $m \times n$ matrix $A$ with entries $a_{i j}$ giving the expected number of times $S_{i}$ needs to retrieve the MDOs in $D_{j}$.

EXAMPLE 1 (continued). Suppose the user navigation probabilities in the three sites are,

$$
\begin{aligned}
& \begin{array}{lllll}
D_{1} & D_{2} & D_{3} & D_{4} & E
\end{array} \\
& D_{1}\left[\begin{array}{lllll}
0.2 & 0.1 & 0.1 & 0.3 & 0.3
\end{array}\right. \\
& D_{2}\left[\begin{array}{ccccc}
0.2 & 0.1 & 0.1 & 0.3 & 0.3 \\
0.1 & 0.2 & 0.1 & 0
\end{array}\right] \\
& P^{1}=D_{3} \quad 0 \quad 0.2 \quad 0.6 \quad 0.1 \quad 0.1 \text {, } \\
& \begin{array}{llllll}
D_{4} & 0.1 & 0.2 & 0.2 & 0.4 & 0.1
\end{array} \\
& \begin{array}{c}
D_{4} \\
E
\end{array}
\end{aligned}
$$

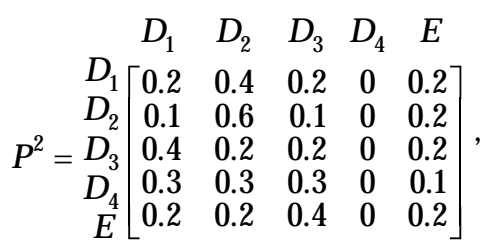

$$
\begin{aligned}
& \begin{array}{lllll}
D_{1} & D_{2} & D_{3} & D_{4} & E
\end{array} \\
& D_{1}\left[\begin{array}{lllll}
0.5 & 0 & 0 & 0.4 & 0.1
\end{array}\right] \\
& \begin{array}{lllllll}
D_{2} & 0.2 & 0.3 & 0.2 & 0.1 & 0.2
\end{array}
\end{aligned}
$$

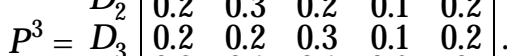

$$
\begin{aligned}
& \begin{array}{c|ccccc}
D_{4} & 0.2 & 0.1 & 0.1 & 0.6 & 0 \\
0.3 & 0.1 & 0.1 & 0.2 & 0.3
\end{array}
\end{aligned}
$$


After the analyses of the long run behavior of these Markov chains, the expected starting document frequencies out of $F=\{900,800,900\}$ browsing sessions, matrix B is,

$$
B=S_{S_{2}} S_{S_{3}}\left[\begin{array}{cccc}
100 & D_{2} & D_{3} & D_{4} \\
S_{3} & 300 & 300 & 200 \\
300 & 400 & 200 & 0 \\
300 & 100 & 400
\end{array}\right] .
$$

Then the matrix $\mathrm{A}(B \times R)$ is,

$$
A=S_{S_{2}}^{S_{3}}\left[\begin{array}{cccc}
D_{1} & D_{2} & D_{3} & D_{4} \\
S_{3} & 340 & 630 & 296 \\
260 & 440 & 580 & 132 \\
515 & 200 & 530 & 448
\end{array}\right] .
$$

Further, we need the size, starting time, duration, and presentation rate (synchronization constraint specification) of each $M D O$ in each hypermedia document. For the last three items, we only need any two of them; the remaining one can be derived from the other two. This information can be obtained from the OCPN specification of MDOs in a hypermedia document.

A box is added at the beginning of each OCPN which represents the delay in starting the presentation of the hypermedia document so as to adhere to the synchronization requirements. The duration of this delay box is related to the browsing site and the sites where the MDOs in the document are allocated. Thus, we use $d_{i j}$ to represent the duration of the delay box when site $S_{i}$ accesses the document $D_{j}$.

An OCPN representation provides the starting time, start $_{j k}$, and duration, $d u r_{j k}$, of each $O_{k}$ in each document $D_{j}$. In addition, the $n \times l$ usage matrix $U$ is generated from the OCPN specifications (if document $D_{j}$ uses MDO $O_{k}$, then $u_{j k}=1$, otherwise, $u_{j k}=0$ ). Then, by multiplying $A$ by $U$, we can estimate the access frequencies of each $M D O$ from each site. Let size $_{k}$ be the size of $M D O O_{k}$.

With this information, we can calculate $d_{i j}$ as follows,

$$
d_{i j}=\max _{\forall k, u_{j k}=1}\left(\frac{\operatorname{size}_{k}}{c_{\text {site }(k) \cdot i}}-d u r_{j k}-\text { start }_{j k}\right)
$$

where $\operatorname{site}(k)$ represents the site where $O_{k}$ is allocated.

We can calculate the values of all $d_{i j}, 1 \leq i \leq m, 1 \leq j \leq n$, by using the above formula. This formula means that the delay is equal to the maximum value of (transmission duration - presentation duration - presentation starting time) for each $M D O$ in the document. When this value is negative, it implies that the transmission time is shorter than the presentation time, as we can start presenting the MDOs in the hypermedia document as soon as the MDOs arrive at the end-user site. When this value is positive, we must delay the presentation, otherwise the MDOs presentation cannot end at the synchronization time, and hence will not adhere to the synchronization constraints.

For example, suppose we need to present the document with OCPN specification shown in Fig. 6. If the MDO $A$ is not in the site where this document is presented, we need to retrieve it from the network. If the transmission duration for the whole MDO $A$ is greater than 30 , we need to introduce some additional delay for fulfilling the synchronization requirement (to avoid jitter).

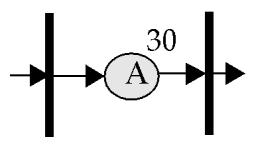

Fig. 6. OCPN representation of a simple MDO.

Therefore, the objective function is,

$$
t=\sum_{i \in S} \sum_{j \in D} d_{i j} \cdot a_{i j}
$$

By minimizing this value through the change of the function site $(k)$, we obtain the data allocation scheme that is optimal (the (delay incurred) response time is minimal), while adhering to the synchronization constraints.

EXAMPLE 1 (continued). There are three MDOs, namely, $A, B$ and $C$ ( $E$ is a delay state, so there is no associative actual $M D O)$. If we allocate $A$ at Site $2, B$ at Site 3 , and $C$ at site 1 , then $d_{11}$ is equal to,

$$
d_{11}=\max \left(\left(\frac{2,280}{38}-15-40\right),\left(\frac{1,220}{41}-55-0\right)\right)=5 \text {. }
$$

Similarly, we can calculate the values of all $d_{i j}, 1 \leq i \leq 3$, $1 \leq j \leq 3$, as we have the MDO allocation scheme. And the total response time delay will be,

$$
11430+40650+29130=81210 .
$$

\subsection{User Interactions and Buffer Space Constraints}

The model presented above does not consider user interactions and buffer space constraints. It assumes that the user does not interrupt the presentation and the size of the local storage facility is large enough for storing any one of the hypermedia documents in the database system. The original OCPN model does not incorporate user interactions. As stated in Section 2, we can model these kinds of user interactions by using the traditional timed-Petri net. However, as there are many different kinds of interactions, this method alone is insufficient. The model must include the semantics for user interactions as well. There are a number of extensions to the Petri net that include these semantics [17], [20], [25]. However, in our paper, we only need to be concerned with the probabilities of each user interaction; therefore, we will not introduce these models here. Interested readers can refer to these papers for details. By including user interactions and buffer space constraints, there can be four different cases for hypermedia document allocation problem given below.

\subsubsection{No User Interaction and Unlimited Buffer Space}

This is essentially the best scenario, because we can retrieve all MDOs in a hypermedia document at the beginning since there is no storage limitation. As there is no user interaction, the data can be discarded immediately after use. The cost function for the response time for each hypermedia document, as presented in Section 3.1, is the maximum of the delays of the embedded MDOs for satisfying the synchronization requirements. 


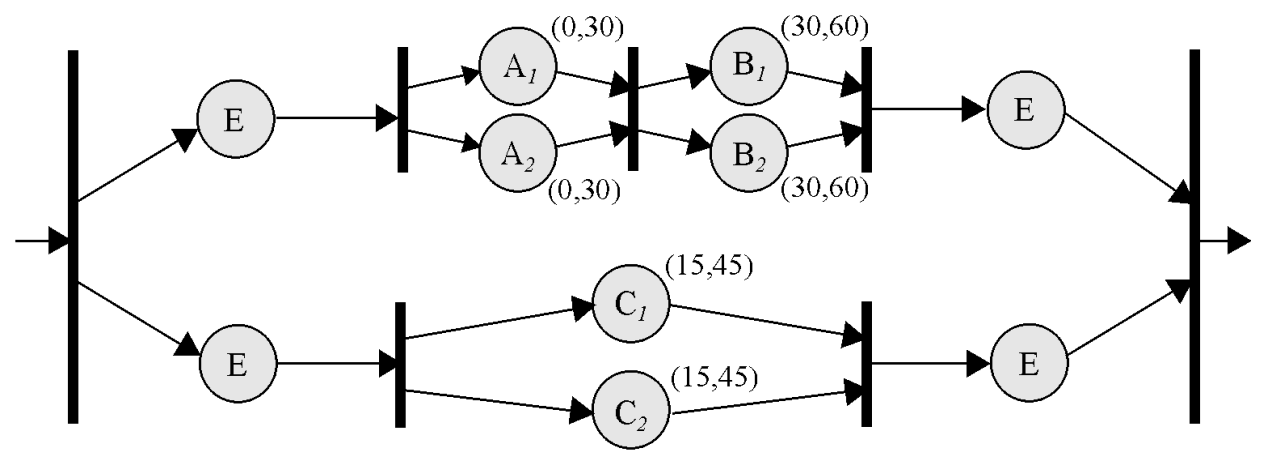

Fig. 7. The OCPN specification of synchronization requirements for document $D_{5}$.

\subsubsection{User Interaction and Unlimited Buffer Space}

By including user interactions, some of the MDOs in a hypermedia document may need to be presented multiple times (e.g., play in reverse or stop and resume later). However, as there is unlimited buffer space, the system can store all MDOs of a hypermedia document once they are retrieved. Therefore, the delay for handling the user interactions is due to some local processing that is irrelevant to the data allocation of the MDOs. The cost function is thus the same as that in the Section 3.1.

\subsubsection{No User Interaction and Limited Buffer Space}

In this scenario, the system cannot retrieve all the MDOs in advance. Instead, the system must retrieve the MDOs only when it needs to present these MDOs. Therefore, every synchronization point in the hypermedia document may cause some delay and the cost function in such a situation is the summation of these delays. Indeed, the model presented in Section 3.1 can be generalized to deal with this scenario.

First, we need to decompose each document into component subdocuments. From the OCPN specifications, we know the states representing the $M D O$ s in each document. Denoting this set of states as $S$ and for $\forall s, s \in S$, we can get the starting time and ending time of the state (i.e., presentation of the corresponding MDO) from the OCPN specifications. Then, we can use the algorithm DECOMPOSE_DOCUMENT(S) to decompose the document into its subdocuments. The algorithm is outlined on the next page.

EXAMPLE 2. Suppose we have a hypermedia document $D_{5}$ shown in Fig. 7. Then, we can decompose it into three subdocuments (at the synchronization points) as shown in Fig. 8.

Denote these subdocuments as $D_{A}, D_{B}$, and $D_{C}$. Calculating the delays of these subdocuments and summing them up will give the total delay of the original document $D_{1}$. The remaining problems of replacing $D_{1}$ by $D_{A}, D_{B}$, and $D_{C}$ are the corresponding updates of the two matrices $R$ and $B$. For the matrix $R$, as $D_{A}$ is the starting subdocument of $D_{1}$, we replace the label of $D_{1}$ by $D_{A}$. After that, we add two columns and two rows for $D_{B}$ and $D_{C}$. If we browse to $D_{A}$, both $D_{B}$ and $D_{C}$ will be retrieved. Therefore, set the values $r_{A B}$ and $r_{A C}$ to 1 . Besides, set $r_{B B}$ and $r_{C C}$ to 1 .
Set the remaining values that are not yet specified to 0 . This is because we will not need other documents if we are in $D_{B}$ or $D_{C}$ and we will not need to retrieve $D_{B}$ or $D_{C}$ in other documents.

The matrix $B$ represents the probability of using each document as the initial browsing document. Thus, we only need to change the label of $D_{1}$ to $D_{A}$ (the starting subdocument) and add two columns representing $D_{B}$ and $D_{C}$. In these two columns, set all the entries to 0 , as we cannot start browsing from $D_{B}$ or $D_{C}$. Thus, by decomposing each hypermedia document at its synchronization points, the model presented in Section 3.1 incorporates the constraints of limited storage.

\subsubsection{User Interaction and Limited Buffer Space}

If we know the expected number of times each subdocument will be presented in each hypermedia document, we can calculate the expected response time of each document in each site, which is just the weighted sum of the delays of the subdocuments in the document. In the previous scenario, the expected number of times each subdocument is needed is 1 , so the cost function is just the summation of the delays. After employing the algorithm DECOMPOSE_DOCUMENT(S) to decompose documents, we do not need to distinguish documents or subdocuments and we will use documents to represent both from now on.

To calculate the expected number of times each document is needed, we must know the probabilities of relevant user interactions. Relevant means that the interaction will need to retrieve the MDOs in the document again such as reverse playing or stop and resume. Other interactions that will not need the retrieval of the MDO again (for instance, termination of browsing) can be ignored. Once we have these probabilities, we can calculate the expected number of times each document is presented by employing the first step analysis method [23]. Note that these probabilities can be generated by observing user interaction over a period of time. Alternatively, we can use Markov chain to model the inter-MDO user interaction and obtain the required probabilities from long run behavior analysis.

For example, suppose the relevant probability of an $M D O O_{k}$ in a document $D_{j}$ is $i p_{j k}$. Assume that the expected 

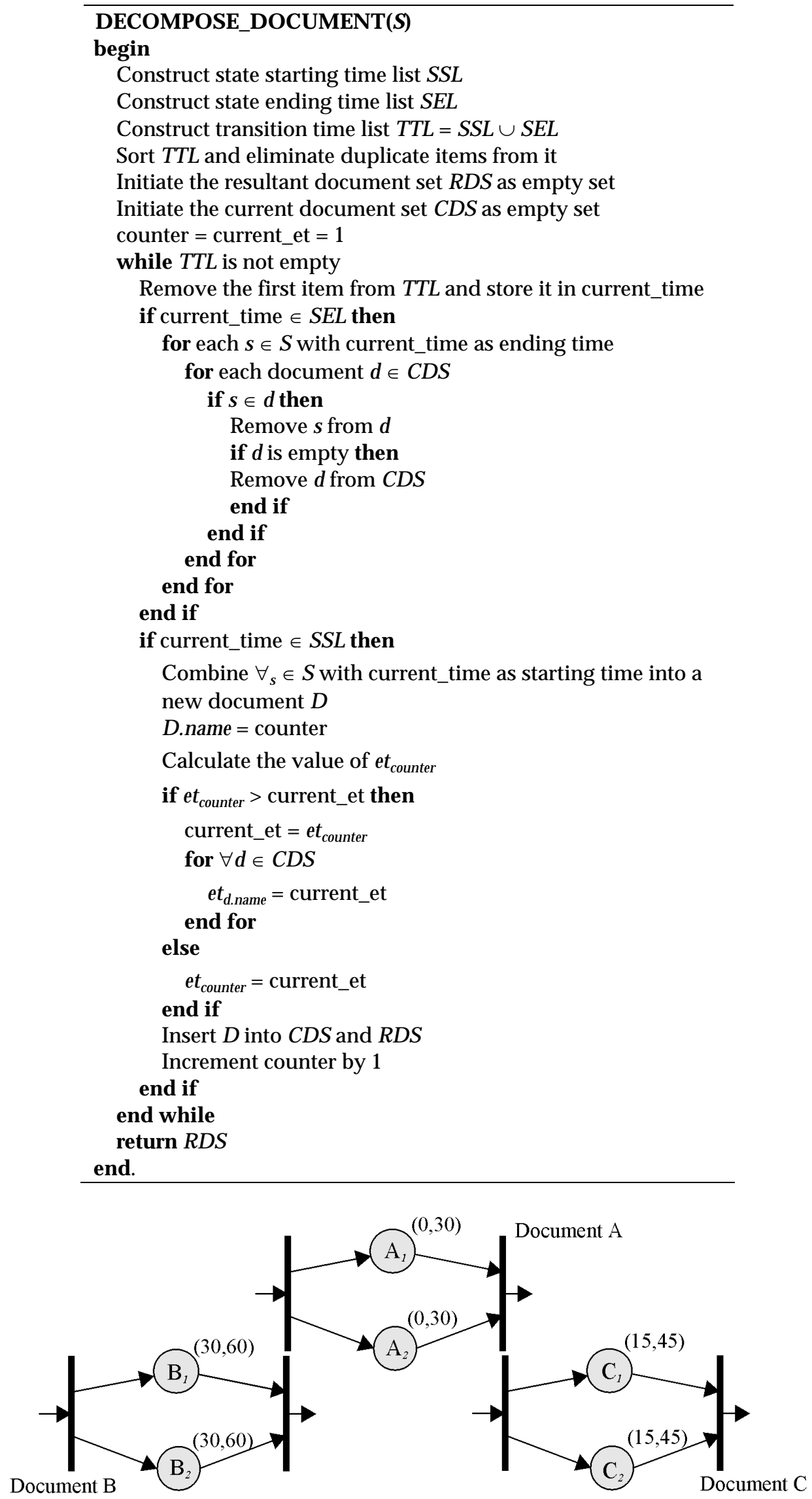

Fig. 8. Subdocuments OCPN specification of synchronization requirements for document $D_{5}$. 


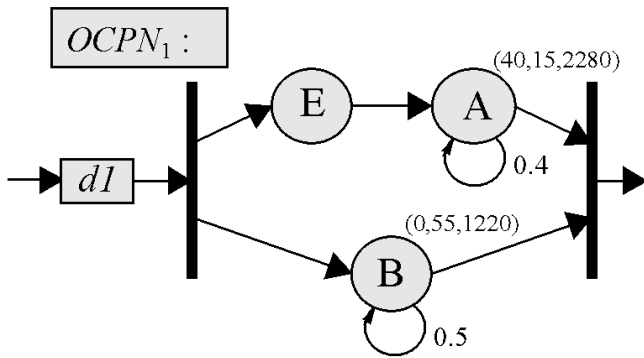

Fig. 9. The augmented OCPN by including user-interaction.

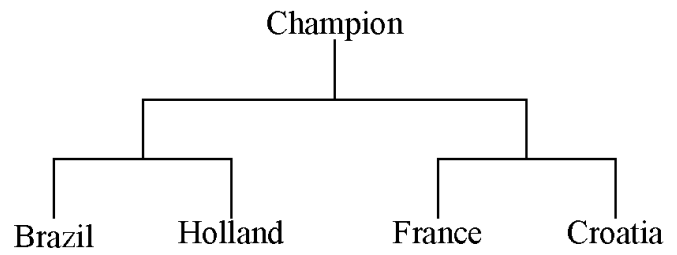

Fig. 10. The scenario for 1998 World Cup semifinal round.

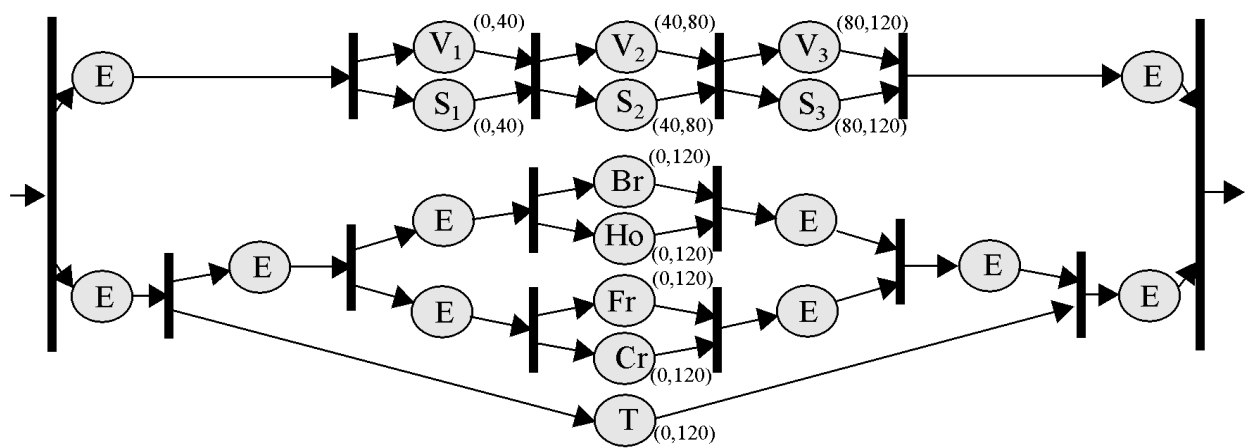

Fig. 11. The 1998 World Cup at semifinal round $\left(D_{1}\right)$.

number of times this MDO is needed is mdo_et $t_{j k}$. Then, we have, ${ }^{1}$

$$
\begin{gathered}
m d o \_e t_{j k}=1+i p_{j k} \times m d o \_e t_{j k} \\
m d o \_e t_{j k}=\left(1-i p_{j k}\right)^{-1} .
\end{gathered}
$$

Similarly, we can estimate the expected number of times other MDOs composing this document are needed. Then, the expected number of times this document is needed is just the maximum of these values. Denoting this value as $e t_{j}$ for document $D_{j}$, we have,

$$
e t_{j}=\max \left(m d o \_e t_{j k}\right), \text { for } \forall k, u_{j k}=1 \text {. }
$$

And the delays $d_{i j}$ will become,

$$
\begin{gathered}
d_{i j}=\left(\max \left(\frac{\text { size }_{k}}{C_{\text {site }(k) \cdot i}}-\text { dur }_{j k}-\text { start }_{j k}\right)\right) \cdot \text { et }_{j}, \\
\text { for } \forall k, u_{j k}=1
\end{gathered}
$$

We have made an assumption here that we can retrieve the $M D O$ s from anywhere in the middle of it. If this assumption is violated, we can only get the whole $M D O$ again even if the user just wants the last part of it. Otherwise, the assumption will introduce some overhead. With uniform distribution, the overhead will be half of the $M D O$ presentation duration. That is,

$$
\text { overhead }_{i j}=\frac{\max \left(\text { dur }_{j k}+\text { start }_{j k}\right) \cdot\left(e t_{j}-1\right)}{2} .
$$

And the cost function will become,

$$
t=\sum_{i \in S} \sum_{j \in D}\left(d_{i j}+\text { overhead }_{i j}\right) \cdot a_{i j} .
$$

Suppose we add user interactions and worst case buffer space constraints to the hypermedia database system in

1. Or $m d o_{-} e t=1+i p_{j k}+i p_{j k}^{2}+\ldots=\left(1-i p_{j k}\right)^{-1}$.
Example 1. After adding the probability of relevant user interruption to the $M D O$, the augmented OCPN of $D_{1}$ is shown in Fig. 9.

Thus, the expected number of times $M D O A$ is needed when document $D_{1}$ is retrieved is given by:

$$
\begin{gathered}
\text { mdo_et } t_{1 A}=1+0.4 \times \text { mdo_et }_{1 A}, \\
\text { mdo_et } t_{1 A}=\frac{1}{1-0.4}=1.667 .
\end{gathered}
$$

Similarly, the expected number of times $M D O B$ is needed is given by:

$$
\begin{gathered}
\text { mdo_et } t_{1 B}=1+0.5 \times \text { mdo_et } t_{1 B}, \\
m d o \_e t_{1 B}=\frac{1}{1-0.5}=2 .
\end{gathered}
$$

Note that when we need $B$ again, $A$ is also needed. Thus, $e t_{1}=\max (1.667,2)=2$.

Since we have the worst-case buffer space constraints, the delay $d_{11}$ will become

$$
d_{11}=\left(\max \left(\left(\frac{2,280}{38}-15-40\right),\left(\frac{1,220}{41}-55-0\right)\right)\right) \times 2=10 .
$$

\subsection{A Real-World Example}

The four semifinalists of the 1998 World Cup Football are Brazil, Holland, France, and Croatia as shown in Fig. 10.

The World Cup hypermedia documents are updated accordingly, there are now five hypermedia documents. One for the overview and four others (one for each team). The hypermedia database system contains three sites, one located in Europe, one in Asia and one in South America. Users will use the site nearest to them for retrieving the most up-to-date information.

The OCPN specifications of the $D_{1}$ and $D_{2}$ are shown in Fig. 11 and Fig. 12, respectively. $D_{3}, D_{4}$, and $D_{5}$ are similar to 


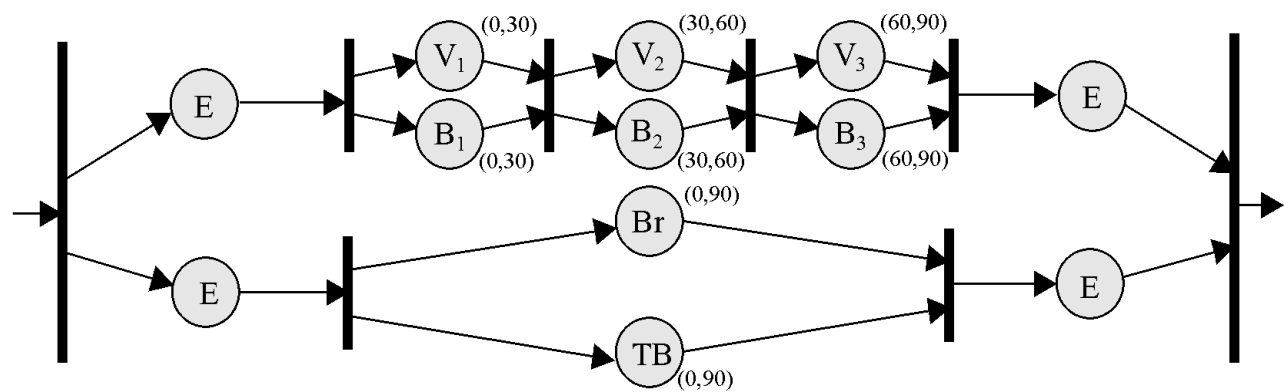

Fig. 12. Information about the Brazilian team (D2).

TABLE 2

DESCRIPTION OF MDOS IN THE HYPERMEDIA DATABASE SYSTEM

\begin{tabular}{|c|c|c|}
\hline Object & Description & Size (in Kilobytes) \\
\hline $\mathrm{V}_{1}$ & \multirow{3}{*}{ Image files, an animation of a football drawn by hand. } & 401 \\
\hline $\mathrm{V}_{2}$ & & 1201 \\
\hline $\mathrm{V}_{3}$ & & 601 \\
\hline $\mathrm{S}_{1}$ & \multirow{3}{*}{ Audio files, recording the 1998 World Cup Song. } & 1501 \\
\hline $\mathrm{S}_{2}$ & & 1501 \\
\hline $\mathrm{S}_{3}$ & & 1501 \\
\hline $\mathrm{Br}$ & Photo of the Brazil football team. & 301 \\
\hline Ho & Photo of the Holland football team. & 301 \\
\hline Fr & Photo of the France football team. & 301 \\
\hline $\mathrm{Cr}$ & Photo of the Croatia football team. & 301 \\
\hline $\mathrm{T}$ & General information about the 1998 World Cup in text format. & 101 \\
\hline $\mathrm{B}_{1}$ & \multirow{3}{*}{ The national anthem of Brazil. } & 1201 \\
\hline $\mathrm{B}_{2}$ & & 1201 \\
\hline $\mathrm{B}_{3}$ & & 1201 \\
\hline TB & Information of the Brazil team in text format. & 201 \\
\hline $\mathrm{H}_{1}$ & \multirow{3}{*}{ The national anthem of Holland. } & 1201 \\
\hline $\mathrm{H}_{2}$ & & 1201 \\
\hline $\mathrm{H}_{3}$ & & 1201 \\
\hline $\mathrm{TH}$ & Information of the Holland team in text format. & 201 \\
\hline $\mathrm{F}_{1}$ & \multirow{3}{*}{ The national anthem of France. } & 1201 \\
\hline $\mathrm{F}_{2}$ & & 1201 \\
\hline $\mathrm{F}_{3}$ & & 1201 \\
\hline TF & Information of the France team in text format. & 201 \\
\hline $\mathrm{C}_{1}$ & \multirow{3}{*}{ The national anthem of Croatia. } & 1201 \\
\hline $\mathrm{C}_{2}$ & & 1201 \\
\hline $\mathrm{C}_{3}$ & & 1201 \\
\hline TC & Information of the Croatia team in text format. & 201 \\
\hline$E$ & Empty placeholder. & 0 \\
\hline
\end{tabular}

$D_{2}$ except that they contain other teams information, $D_{3}$ for Holland, $D_{4}$ for France, and $D_{5}$ for Croatia. The size and content of the MDOs are shown in Table 2.

The three sites of the hypermedia database system are fully connected, with the network transmission speed (in kilobytes per second) between them shown in Table 3.
TABLE 3

Transmission SpeEd BetWeEn the THReE Sites (IN KILOBYTES PER SECOND)

\begin{tabular}{|c|c|c|c|}
\cline { 2 - 4 } \multicolumn{1}{c|}{} & Asia & Europe & South America \\
\hline \hline Asia & 0 & 15 & 18 \\
\hline Europe & 15 & 0 & 10 \\
\hline South America & 18 & 10 & 0 \\
\hline
\end{tabular}


The probability matrix of browsing from a document to another document is,

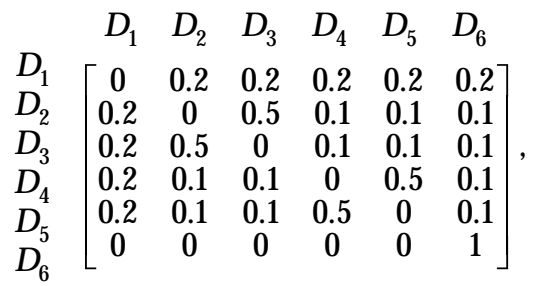

where $D_{6}$ represents the end of the browsing session.

Using the methodology developed in Section 3 with $b p l$ of the trees limited to 0.01 , we have the following $5 \times 5$ matrix, $R$, representing the probability of retrieving MDOs in a document if we start browsing from a specific document,

$$
\begin{aligned}
& \begin{array}{lllll}
D_{1} & D_{2} & D_{3} & D_{4} & D_{5}
\end{array} \\
& D_{1}\left[\begin{array}{ccccc}
1 & 0.2 & 0.2 & 0.2 & 0.2
\end{array}\right] \\
& \left.\begin{array}{llllll}
D_{2} & 0.2 & 1 & 0.5 & 0.1 & 0.1
\end{array}\right] \text {. } \\
& \begin{array}{l|lllll}
D_{3} & 0.2 & 0.5 & 1 & 0.1 & 0.1
\end{array} \\
& \begin{array}{c|ccccc}
D_{4} & 0.2 & 0.1 & 0.1 & 1 & 0.5 \\
D_{5} & 0.2 & 0.1 & 0.1 & 0.5 & 1
\end{array}
\end{aligned}
$$

The users behavior in the three sites are different. The European and South American users are more interested in the teams representing their region. The Asian users do not have such a bias. However, Brazil is the favorite team in

\begin{tabular}{|c|c|c|c|c|c|}
\hline$D_{1}$ & $D_{2}$ & $D_{3}$ & $D_{4}$ & $D_{5}$ & $N$ \\
\hline 0.3 & 0.2 & 0.1 & 0.1 & 0.1 & $0.2]$ \\
\hline$D_{2}$ & 0.0 & 0.2 & 0.1 & 0.1 & \\
\hline 0.2 & 0.2 & 0.2 & 0.1 & 0.1 & 0.2 \\
\hline 0.2 & 0.2 & 0.1 & 0.2 & 0.2 & 0.1 \\
\hline 0.2 & 0.2 & 0.1 & 0.2 & 0.2 & 0.1 \\
\hline 0.4 & 0.3 & 0 & 0 & 0 & 0.3 \\
\hline
\end{tabular}
Asia and $D_{2}$ is accessed more often than other documents. The following gives the transition matrices for the initial browsing document for the three sites.

In Asia $\left(P^{1}\right)$,

\begin{tabular}{|c|c|c|c|c|c|}
\hline$D_{1}$ & $D_{2}$ & $D_{3}$ & $D_{4}$ & $D_{5}$ & $N$ \\
\hline 0.2 & 0.1 & 0.1 & 0.2 & 0.2 & 0.27 \\
\hline & 0.2 & 0.1 & 0.2 & & 0.1 \\
\hline 0.2 & 0.1 & 0.2 & 0.2 & 0.2 & 0.1 \\
\hline 0.1 & 0.1 & 0.1 & 0.3 & 0.3 & 0.1 \\
\hline 0.1 & 0.1 & 0.1 & 0.3 & 0.3 & 0.1 \\
\hline 0.3 & 0 & 0 & 0.3 & 0.3 & 0.1 \\
\hline
\end{tabular}

where $N$ represents the not-start-browsing in the next

\begin{tabular}{|c|c|c|c|c|c|}
\hline$D_{1}$ & $D_{2}$ & $D_{3}$ & $D_{4}$ & $D_{5}$ & $N$ \\
\hline 0.2 & 0.2 & 0.2 & 0.1 & 0.1 & 0.2 \\
\hline & 0.3 & 0.3 & 0.1 & 0.1 & 0.1 \\
\hline$D_{3}$ & 0.3 & 0.3 & 0.1 & 0.1 & 0.1 \\
\hline 0.2 & 0.2 & 0.2 & 0.2 & 0.1 & 0.1 \\
\hline 0.2 & 0.2 & 0.2 & 0.1 & 0.2 & 0.1 \\
\hline 0.3 & 0.3 & 0.3 & 0 & 0 & 0.1 \\
\hline
\end{tabular}
transition.

The user behavior in Europe $\left(P^{2}\right)$ is,

Correspondingly, that in South America $\left(P^{3}\right)$ is,
In the long run, the probability matrix of the initial browsing document at each site, $B$, is ( $F$ is set to $\{1,000$, $1,000,1,000\})$,

$$
\begin{array}{rrrrr}
D_{1} & D_{2} & D_{3} & D_{4} & D_{5} \\
S_{1} \\
S_{2} \\
S_{3}
\end{array}\left[\begin{array}{rrrrr}
317 & 293 & 146 & 122 & 122 \\
182 & 114 & 114 & 295 & 295 \\
182 & 295 & 295 & 114 & 114
\end{array}\right] .
$$

Denote the first row of this row as $x$. We can get $x$ from $P^{1}$ by solving the linear equations, (similarly, use $P^{2}$ for row 2 and $P^{3}$ for row 3 )

$$
x \cdot\left(P^{1}\right)^{\mathrm{T}}=x
$$

where $\mathrm{A}^{\mathrm{T}}$ is the transpose of matrix $\mathrm{A}$.

Discard the long run probability of not browsing and normailize the remaining probabilities, we will get the probability of using each document as entry point for every browsing session. Multiply these values with expected

\begin{tabular}{|c|c|c|c|c|}
\hline$D_{1}$ & $D_{2}$ & $D_{3}$ & $D_{4}$ & $D_{5}$ \\
\hline 454 & 454 & 380 & 290 & 290 \\
\hline 345 & 266 & 266 & 502 & 502 \\
\hline 345 & 502 & 502 & 266 & 266 \\
\hline
\end{tabular}
number of browsing sessions initiated in unit time, we will have the access frequencies of each document as initial browsing document in unit time.

By multiplying $B$ matrix by the matrix of expected navigation path $(R)$, we get the expected access frequency matrix for retrieving each document from each site in a period of time, $A$,

From this matrix and from the OCPN specifications of each document, we can estimate the expected number of times each $M D O$ is retrieved from each site, $A \times U$ (see Table 4).

The delay of the multimedia presentation of the document $D_{j}$ in site $S_{i}$ will be,

$$
d_{i j}=\max \left(\frac{\operatorname{size}_{k}}{c_{\text {site }(k) \cdot i}}-d u r_{j k}-s_{\text {sart }} t_{k}\right), \text { for } \forall k, u_{j k}=1 .
$$

Suppose we allocate the MDOs randomly to the sites, for example, allocate $O_{i}$ at site $(i \bmod 3)$. The presentation delay of each document in each site is (in seconds),

\begin{tabular}{|c||c|c|c|}
\cline { 2 - 4 } \multicolumn{1}{c|}{} & Site 1 & Site 2 & Site 3 \\
\hline \hline Document 1 & 20.1 & 60.1 & 70.1 \\
\hline Document 2 & 36.7 & 90.1 & 60.1 \\
\hline Document 3 & 20.1 & 30.1 & 60.1 \\
\hline Document 4 & 50.1 & 60.1 & 90.1 \\
\hline Document 5 & 36.7 & 90.1 & 60.1 \\
\hline
\end{tabular}

Using the expected number of retrievals for each document from each site, we can calculate the expected total delay in each site as,

\begin{tabular}{|c|c|c|c|}
\cline { 2 - 4 } \multicolumn{1}{c|}{} & Site 1 & Site 2 & Site 3 \\
\hline \hline Delay & 58,597 & 128,108 & 124,548 \\
\hline
\end{tabular}

The total expected delay of the whole system is 311,313 seconds. 
TABLE 4

EXPECTEd RETRIEVAL FREQUENCIES OF MDOS FROM EACH Site

\begin{tabular}{|c|c||c|c|c|}
\hline Media Item & Object & Site 1 & Site 2 & Site 3 \\
\hline \hline $\mathrm{O}_{1}$ & $\mathrm{~V}_{1}$ & 1868.29 & 1881.82 & 1881.82 \\
\hline $\mathrm{O}_{2}$ & $\mathrm{~V}_{2}$ & 1868.29 & 1881.82 & 1881.82 \\
\hline $\mathrm{O}_{3}$ & $\mathrm{~V}_{3}$ & 1868.29 & 1881.82 & 1881.82 \\
\hline $\mathrm{O}_{4}$ & $\mathrm{~S}_{1}$ & 453.66 & 345.45 & 345.45 \\
\hline $\mathrm{O}_{5}$ & $\mathrm{~S}_{2}$ & 453.66 & 345.45 & 345.45 \\
\hline $\mathrm{O}_{6}$ & $\mathrm{~S}_{3}$ & 453.66 & 345.45 & 345.45 \\
\hline $\mathrm{O}_{7}$ & $\mathrm{Br}$ & 907.32 & 611.36 & 847.73 \\
\hline $\mathrm{O}_{8}$ & $\mathrm{Ho}$ & 834.15 & 611.36 & 847.73 \\
\hline $\mathrm{O}_{9}$ & $\mathrm{Fr}$ & 743.90 & 847.73 & 611.36 \\
\hline $\mathrm{O}_{10}$ & $\mathrm{Cr}$ & 743.90 & 847.73 & 611.36 \\
\hline $\mathrm{O}_{11}$ & $\mathrm{~T}$ & 453.66 & 345.45 & 345.45 \\
\hline $\mathrm{O}_{12}$ & $\mathrm{~B}_{1}$ & 453.66 & 265.91 & 502.27 \\
\hline $\mathrm{O}_{13}$ & $\mathrm{~B}_{2}$ & 453.66 & 265.91 & 502.27 \\
\hline $\mathrm{O}_{14}$ & $\mathrm{~B}_{3}$ & 453.66 & 265.91 & 502.27 \\
\hline $\mathrm{O}_{15}$ & $\mathrm{~TB}$ & 453.66 & 265.91 & 502.27 \\
\hline $\mathrm{O}_{16}$ & $\mathrm{H}_{1}$ & 380.49 & 265.91 & 502.27 \\
\hline $\mathrm{O}_{17}$ & $\mathrm{H}_{2}$ & 380.49 & 265.91 & 502.27 \\
\hline $\mathrm{O}_{18}$ & $\mathrm{H}_{3}$ & 380.49 & 265.91 & 502.27 \\
\hline $\mathrm{O}_{19}$ & $\mathrm{TH}$ & 380.49 & 265.91 & 502.27 \\
\hline $\mathrm{O}_{20}$ & $\mathrm{~F}_{1}$ & 290.24 & 502.27 & 265.91 \\
\hline $\mathrm{O}_{21}$ & $\mathrm{~F}_{2}$ & 290.24 & 502.27 & 265.91 \\
\hline $\mathrm{O}_{22}$ & $\mathrm{~F}_{3}$ & 290.24 & 502.27 & 265.91 \\
\hline $\mathrm{O}_{23}$ & $\mathrm{TF}$ & 290.24 & 502.27 & 265.91 \\
\hline $\mathrm{O}_{24}$ & $\mathrm{C}_{1}$ & 290.24 & 502.27 & 265.91 \\
\hline $\mathrm{O}_{25}$ & $\mathrm{C}_{2}$ & 290.24 & 502.27 & 265.91 \\
\hline $\mathrm{O}_{26}$ & $\mathrm{C}_{3}$ & 290.24 & 502.27 & 265.91 \\
\hline $\mathrm{O}_{27}$ & $\mathrm{TC}$ & 290.24 & 502.27 & 265.91 \\
\hline & & & & \\
\hline
\end{tabular}

Now, suppose we allocate the data differently, place MDOs $O_{1}$ to $O_{11}$ in Asia, $O_{12}$ to $O_{19}$ in South America and others in Europe. The presentation delay will become,

\begin{tabular}{|c||c|c|c|}
\cline { 2 - 4 } \multicolumn{1}{c|}{} & Site 1 & Site 2 & Site 3 \\
\hline \hline Document 1 & 0 & 60.1 & 43.4 \\
\hline Document 2 & 36.7 & 90.1 & 0.1 \\
\hline Document 3 & 36.7 & 90.1 & 0.1 \\
\hline Document 4 & 50.1 & 0.1 & 90.1 \\
\hline Document 5 & 50.1 & 0.1 & 90.1 \\
\hline
\end{tabular}

And the expected total time delay in each site will be,

\begin{tabular}{|c|c|c|c|}
\cline { 2 - 4 } \multicolumn{1}{c|}{} & Site 1 & Site 2 & Site 3 \\
\hline \hline Delay & 59,666 & 68,828 & 63,050 \\
\hline
\end{tabular}

The total expected delay of the whole system in this case is 191,544 seconds, with 38 percent reduction in the response time. In a system with three sites and five hypermedia documents, such performance improvement shows that good data allocation schemes are critically needed.

\section{Proposed Data Allocation Algorithms}

The data allocation problem in its simple form has been shown to be NP-complete [4] and the problem discussed here is more complex than the simple case; there are $k^{m}$ different allocation schemes for a system with $m$ sites and $k$ $M D O$ s, implying that an exhaustive search would require $O\left(k^{m}\right)$ in the worst case to find the optimal solution. Therefore, we must use heuristic algorithms to solve the problem.

\subsection{The Hill-Climbing Approach}

We have developed an algorithm based on the Hill-Climbing technique to find a near optimal solution. The data allocation problem solution consists of the following two steps:

1) Find an initial solution.

2) Iteratively improve the initial data allocation by using the hill climbing heuristic until no further reduction in total response time can be achieved. This is done by applying some operations on the initial allocation scheme. Since there are finite number of allocation schemes, the heuristic algorithm will complete its execution. 


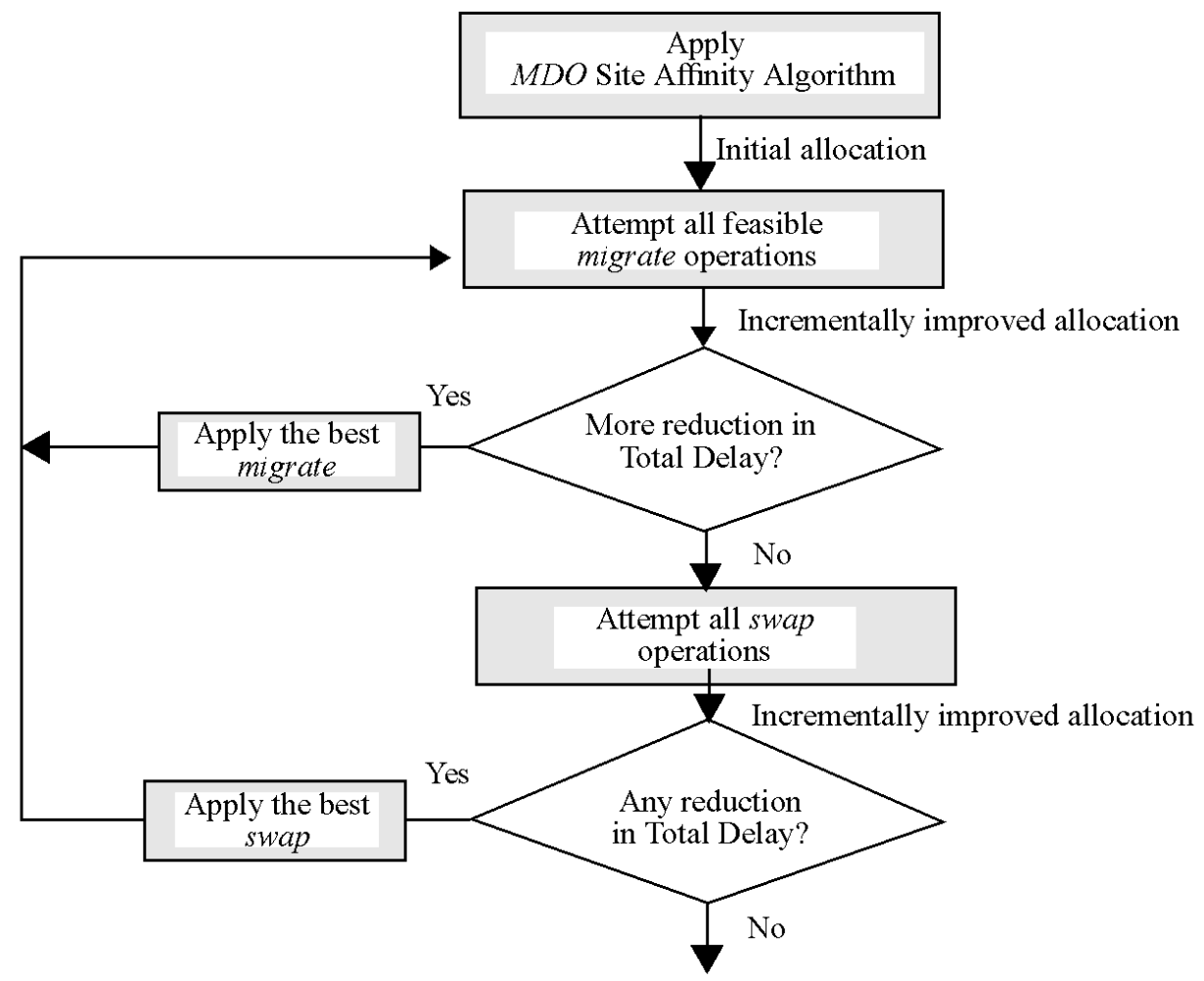

Final Allocation

Fig. 13. Steps in the Hill-Climbing algorithm for data allocation problem.

For step one, one possibility is to obtain the initial solution by allocating the MDOs to the sites randomly. However, a better initial solution can be generated by allocating an $M D O$ to the site which retrieves it most frequently (this information can be obtained from the matrix $A \times U$ ). If that site is already saturated, we allocate the $M D O$ to the next site that needs it the most. We call this method the MDO site affinity algorithm.

In the second step, we apply some operations on the initial solution to reduce the total response time. Two types of operations are defined, namely

- migrate (move MDOs from its currently allocated site to another site), and

- swap (swap the locations of one set of MDOs with the locations of another set of $M D O s$ ).

These operations are iteratively applied until no more reduction is observed in the total response time. Fig. 13 shows the major steps in the Hill-Climbing heuristic algorithm.

The set of migrate and swap operations are as follows.

- migrate $\left(O_{j}, S_{i}\right)$ : Move $M D O O_{j}$ to $S_{i}$. This operation can be applied to each $M D O$, and an $M D O$ can be moved to any one of the $m-1$ sites at which it is not located. Therefore, there can be a maximum of $k(m-1)$ migrate operations that can be applied during each iteration.

- $\operatorname{swap}\left(O_{x}, O_{x^{\prime}}\right)$ : Swap the location of $M D O O_{x}$ with the location of $M D O O_{x}$. This operation can be applied to each distinct pair of MDOs. Therefore, there can be a maximum of $k(k-1) / 2$ swap operations that can be applied during each iteration. Although this operation is equivalent to two migrate operations, it is necessary as some of the sites may be already saturated such that we cannot migrate MDO to it any more.

Here, we apply the Hill-Climbing algorithm to the example presented in Section 3.1. First, we calculate the matrix $A \times U$ for finding the initial allocation solution:

$$
A \times U=\left[\begin{array}{rrr}
585 & 1,171 & 1,266 \\
700 & 972 & 1,152 \\
715 & 1,493 & 1,178
\end{array}\right] .
$$

It is easy to see that the initial allocation is $\left\{S_{3}, S_{3}, S_{1}\right\}$. Then, the total response time given by this initial allocation is $8,860+43,280++23,910=76,050$.

Table 5 shows the migrate and swap operations applied to improve the initial solution provided by the $M D O$ site affinity algorithm. For example, after applying the migrate $\left(O_{1}, S_{1}\right)$, the total response time delay reduces from 76,050 to 66,170 . The solution to the data allocation problem generated by the Hill-Climbing algorithm is $\left\{S_{1}, S_{3}, S_{1}\right\}$ (i.e., $M D O A$ is allocated at site $S_{1}, M D O B$ at site $S_{3}$, and $M D O C$ at site $S_{1}$ ) with the total response time incurred to execute the query being equal to 66,170 . Table 6 shows all of the feasible allocation schemes and the total response time incurred for each of them.

Comparing the Hill-Climbing algorithm with the exhaustive solution, we observe that the Hill-Climbing algorithm can generate the optimal solution (which is allocation number 7 in Table 6). However, the Hill-Climbing algorithm does not guarantee an optimal solution. 
TABLE 5

OUtPUt OF THE HILL-CLIMBING ALGORITHM FOR DATA ALLOCATION

\begin{tabular}{|c|c||c|c|c|}
\hline Current Allocation & Operation Type & Operation Applied & New Allocation & Total Response Time Delay \\
\hline \hline$\left\{S_{3}, S_{3}, S_{1}\right\}$ & - & Initial solution & - & 76050 \\
\hline$\left\{S_{3}, S_{3}, S_{1}\right\}$ & migrate & migrate $\left(O_{1}, S_{1}\right)$ & $\left\{S_{1}, S_{3}, S_{1}\right\}$ & 66170 \\
\hline$\left\{S_{1}, S_{3}, S_{1}\right\}$ & migrate & None applied & - & - \\
\hline$\left\{S_{1}, S_{3}, S_{1}\right\}$ & swap & None applied & - & - \\
\hline
\end{tabular}

TABLE 6

Enumeration of All Allocation Schemes and Their Respective Response Time Delays

\begin{tabular}{|c|c|c|c|c|c|}
\hline \multirow{2}{*}{$\begin{array}{c}\text { Allocation } \\
\text { Number }\end{array}$} & \multirow{2}{*}{$\begin{array}{c}\text { Allocation } \\
\text { Scheme } \\
\end{array}$} & \multicolumn{3}{|c|}{ Total Delay in each Site } & \multirow{2}{*}{$\begin{array}{r}\text { Total } \\
\text { Delay }\end{array}$} \\
\hline & & $S_{1}$ & $S_{2}$ & $S_{3}$ & \\
\hline$\overline{1}$ & $\overline{\left\{S_{1}, S_{1}, S_{1}\right\}}$ & $\overline{0}$ & 441940 & 24230 & $\overline{66170}$ \\
\hline 2 & $\left\{S_{1}, S_{1}, S_{2}\right\}$ & 37870 & 14500 & 32990 & 85360 \\
\hline 3 & $\left\{S_{1}, S_{1}, S_{3}\right\}$ & 32510 & 48540 & 5450 & 86500 \\
\hline 4 & $\left\{S_{1}, S_{2}, S_{1}\right\}$ & 10 & 41940 & 32990 & 74940 \\
\hline 5 & $\left\{S_{1}, S_{2}, S_{2}\right\}$ & 37870 & 14500 & 32360 & 84730 \\
\hline 6 & $\left\{S_{1}, S_{2}, S_{3}\right\}$ & 32510 & 48540 & 5450 & 86500 \\
\hline 7 & $\left\{S_{1}, S_{3}, S_{1}\right\}$ & 10 & 41940 & 24220 & 66170 \\
\hline 8 & $\left\{S_{1}, S_{3}, S_{2}\right\}$ & 37870 & 14500 & 32990 & 85360 \\
\hline 9 & $\left\{S_{1}, S_{3}, S_{3}\right\}$ & 32520 & 48540 & 5440 & 86500 \\
\hline 10 & $\left\{S_{2}, S_{1}, S_{1}\right\}$ & 11438 & 40650 & 29130 & 81210 \\
\hline 11 & $\left\{S_{2}, S_{1}, S_{2}\right\}$ & 39090 & 10 & 37900 & 77000 \\
\hline 12 & $\left\{S_{2}, S_{1}, S_{3}\right\}$ & 33730 & 47250 & 12260 & 93240 \\
\hline 13 & $\left\{S_{2}, S_{2}, S_{1}\right\}$ & 11430 & 40640 & 29140 & 81210 \\
\hline 14 & $\left\{S_{2}, S_{2}, S_{2}\right\}$ & 39090 & 0 & 37900 & 76990 \\
\hline 15 & $\left\{S_{2}, S_{2}, S_{3}\right\}$ & 33730 & 47240 & 12260 & 93230 \\
\hline 16 & $\left\{S_{2}, S_{3}, S_{1}\right\}$ & 11430 & 40650 & 29130 & 81210 \\
\hline 17 & $\left\{S_{2}, S_{3}, S_{2}\right\}$ & 39090 & 10 & 37900 & 77000 \\
\hline 18 & $\left\{S_{2}, S_{3}, S_{3}\right\}$ & 33730 & 47250 & 5230 & 86210 \\
\hline 19 & $\left\{S_{3}, S_{1}, S_{1}\right\}$ & 160 & 43280 & 23920 & 67360 \\
\hline 20 & $\left\{S_{3}, S_{1}, S_{2}\right\}$ & 38020 & 18100 & 32690 & 88100 \\
\hline 21 & $\left\{S_{3}, S_{1}, S_{3}\right\}$ & 32660 & 49880 & 20 & 82560 \\
\hline 22 & $\left\{S_{3}, S_{2}, S_{1}\right\}$ & 8860 & 43280 & 23930 & 76070 \\
\hline 23 & $\left\{S_{3}, S_{2}, S_{2}\right\}$ & 38020 & 18100 & 32690 & 88810 \\
\hline 24 & $\left\{S_{3}, S_{2}, S_{3}\right\}$ & 32660 & 49890 & 30 & 82580 \\
\hline 25 & $\left\{S_{3}, S_{3}, S_{1}\right\}$ & 8860 & 43280 & 23910 & 76050 \\
\hline 26 & $\left\{S_{3}, S_{3}, S_{2}\right\}$ & 38020 & 18100 & 50770 & 106890 \\
\hline 27 & $\left\{S_{3}, S_{3}, S_{3}\right\}$ & 32660 & 49880 & 0 & 82540 \\
\hline
\end{tabular}

\subsection{The Neighborhood Search Approach}

One drawback of the Hill-Climbing approach is its high complexity. Another problem is that the algorithm can be trapped in some local minimum. This is because the exchange or migration of MDO is done only if the movement will give a better solution. To increase the chances of finding the global optimal solution, we must introduce some probabilistic jumps [11]. The probabilistic jumps must be large enough by involving MDOs that can have a great effect on the solution quality. Otherwise, if the jump is small, the algorithm may remain trapped in the same local minimum. Thus, before the executing the algorithm, we must determine which subset of the MDO set is important.

One possible set of important MDOs are the MDOs that are presented at the beginning of some hypermedia document. The reason being that when we browse a hypermedia document, we must retrieve and use the starting MDO immediately, so their transmission delay will have a great effect on the overall document presentation delay. Thus, we have two sets of MDOs: critical MDOs and noncritical MDOs.

Based on the above discussion, the neighborhood search algorithm is designed as follows,

(1) Get the initial allocation scheme use $M D O$ site affinity algorithm (see Section 4.1);

(2) Construct the two list of critical MDO (CMDO) and other $M D O(O M D O)$

(3) BestRes = infinity; searchcount $=0$;

(4) repeat

(5) searchstep $=0$; counter $=0$;

(6) do $\{/ *$ neighborhood search * /

(7) Choose an OMDO randomly and migrate it to a random site;

(8) Choose two OMDOs and swap them;

(9) Compare the two resultant response times and select the better one; 
(10)

(24) $\}$ while (searchstep $++<$ MAXSTEP and counter $<$ MARGIN);

(25) if BestRes > ResTime(NewScheme) then

(26) BestScheme = NewScheme;

(27) $\quad$ BestRes = ResTime (NewScheme);

(28) endif

The random algorithm starts with an initial solution using the site affinity algorithm and then constructs two lists of MDOs. It then tries to merge OMDOs to some random sites by using either the migrate operation or the swap operation whichever gives more improvement in the solution quality. It continues to do so for MAXSTEP times but will stop if there is no improvement in MARGIN number of trials. It then chooses one of the CMDOs and migrates it to a random site or swap it with another randomly selected CMDO. This continues for MAXCOUNT times. The algorithm preserves the best solution found so far and then performs a neighborhood search on CMDOs again for further improvement.

The worst-case running time of the algorithm is

$$
\text { O(MAXSTEP } \times \text { MAXCOUNT }) \text {. }
$$

It is reasonable to set $M A X S T E P$ as a multiple of the number of OMDOs and the number of sites. Similarly, MAXCOUNT is set to be a multiple of the number of $C M D O$ s and the number of sites.

$$
\begin{gathered}
\text { MAXSTEP }=a \cdot\langle m \cdot| \text { OMDOs }|\rangle \\
\text { MAXCOUNT }=b \cdot\langle m \cdot| \text { CMDOs }|\rangle
\end{gathered}
$$

With these assumptions, we will have an $O\left(m^{2} k^{2}\right)$ algorithm.

\section{RESULtS}

In this section, we present the experimental results for the data allocation algorithms described in the previous sections. Comparisons among these algorithms will be made by considering the quality of solutions and the algorithm running times.

\subsection{Workload}

The example considered in the previous section was used for illustrating how the Hill-Climbing algorithm works. But it had only four documents, three MDOs and three sites and thus only 27 different allocation schemes. Since the solutions were to be compared with the optimal solutions generated by an exhaustive search which takes a large amount of time to experiment for a distributed database system even with moderate number of sites and MDOs (for $k M D O s$ and $m$ sites, there are $k^{m}$ allocation schemes, and for each allocation scheme the total response time needs to be calculated), the problem sizes of the experiments we conducted were limited.

We conducted 25 experiments with the number of $M D O$ s ranging from four to eight, and the number of sites ranging from four to eight. Each experiment consisted of 100 allocation problems with the number of sites and the number of MDOs fixed. Each allocation problem had between four and 16 documents, and each document used a subset of the MDOs with its own temporal constraints on them. The communication network, the $M D O$ sizes, the link costs, and the temporal constraints between $M D O$ s in each document were randomly generated from a uniform distribution. The two data allocation algorithms described above were tested for every case and statistics were collected.

\subsection{Comparison of Allocation Costs}

In Table 7 and Table 8, for each of the experiments conducted in a columnwise fashion, we list the following:

1) the number of MDOs,

2) the number of sites,

3) the number of problems,

4) the number of problems for which the algorithm generated the optimal solution,

5) the average percentage deviation from the optimal solution for those allocations for which the algorithm did not generate optimal solution,

6) the number of near optimal solutions with deviation of less than 5 percent,

7) the number of near optimal solutions with deviation of 5 percent or more but less than 10 percent,

8) the number of near optimal solutions with deviation of 10 percent or more but less than 20 percent, and

9) the number of near optimal solutions with deviation of 20 percent or more.

From Table 7, we note that the Hill-Climbing algorithm generated optimal solutions for a large number of problems: 2,173 cases out of a total of 2,500 cases, corresponding to about 87 percent of the test cases. Most of the nonoptimal solutions are in the range of $0-5$ percent deviation from the optimal solution while a few solutions are in the range of equal to or more than 20 percent. The average percentage (only for nonoptimal cases) is about 9.1557 across all cases. These results indicate that the Hill-Climbing algorithm is able to generate high quality solutions.

Table 8 summarizes the results of the random search algorithm. Compared to the Hill-Climbing algorithm, the 
TABLE 7

EXPeRimental Results OF THE HILL-Climbing Algorithm

\begin{tabular}{|c|c|c|c|c|c|c|c|c|}
\hline \multirow[t]{2}{*}{ No. of Sites } & \multirow{2}{*}{$\begin{array}{l}\text { No. of } \\
M D O \mathrm{~s}\end{array}$} & \multirow{2}{*}{$\begin{array}{c}\text { No.of } \\
\text { Problems }\end{array}$} & \multirow{2}{*}{$\begin{array}{c}\text { No. of } \\
\text { Opt. Sol. }\end{array}$} & \multirow{2}{*}{$\begin{array}{c}\text { Aver: \% } \\
\text { Deviation }\end{array}$} & \multicolumn{4}{|c|}{ Number of Sol. with deviation in range } \\
\hline & & & & & $(0,5 \%)$ & {$[5,10 \%)$} & {$[10,20 \%)$} & {$[20 \%,-)$} \\
\hline 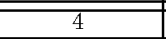 & 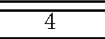 & 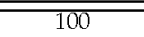 & 93 & $\overline{15.42}$ & 3 & 2 & 0 & 2 \\
\hline 4 & 5 & 100 & 91 & 25.08 & 2 & 1 & 1 & 5 \\
\hline 4 & 6 & 100 & 91 & 8.64 & 5 & 0 & 3 & 1 \\
\hline 4 & 7 & 100 & 90 & 9.23 & 7 & 1 & 0 & 2 \\
\hline 4 & 8 & 100 & 81 & 5.37 & 10 & 7 & 1 & 1 \\
\hline 5 & 4 & 100 & 93 & 15.42 & 3 & 2 & 0 & 2 \\
\hline 5 & 5 & 100 & 91 & 25.08 & 2 & 1 & 1 & 5 \\
\hline 5 & 6 & 100 & 91 & 8.64 & 5 & 0 & 3 & 1 \\
\hline 5 & 7 & 100 & 90 & 9.23 & 7 & 1 & 0 & 2 \\
\hline 5 & 8 & 100 & 81 & 5.37 & 10 & 7 & 1 & 1 \\
\hline 6 & 4 & 100 & 92 & 19.36 & 3 & 1 & 0 & 4 \\
\hline 6 & 5 & 100 & 89 & 6.30 & 7 & 1 & 2 & 1 \\
\hline 6 & 6 & 100 & 84 & 12.59 & 6 & 3 & 4 & 3 \\
\hline 6 & 7 & 100 & 83 & 4.40 & 11 & 3 & 2 & 1 \\
\hline 6 & 8 & 100 & 83 & 11.54 & 13 & 1 & 1 & 2 \\
\hline 7 & 4 & 100 & 97 & 5.23 & 1 & 2 & 0 & 0 \\
\hline 7 & 5 & 100 & 90 & 7.31 & 7 & 1 & 1 & 1 \\
\hline 7 & 6 & 100 & 79 & 8.74 & 15 & 2 & 2 & 2 \\
\hline 7 & 7 & 100 & 85 & 4.41 & 10 & 4 & 1 & 0 \\
\hline 7 & 8 & 100 & 82 & 11.66 & 9 & 3 & 3 & 3 \\
\hline 8 & 4 & 100 & 88 & 3.06 & 9 & 3 & 0 & 0 \\
\hline 8 & 5 & 100 & 87 & 4.81 & 12 & 0 & 0 & 1 \\
\hline 8 & 6 & 100 & 84 & 11.20 & 10 & 2 & 3 & 1 \\
\hline 8 & 7 & 100 & 81 & 8.79 & 9 & 5 & 2 & 3 \\
\hline 8 & 8 & 100 & 77 & 4.00 & 18 & 2 & 2 & 1 \\
\hline
\end{tabular}

TABLE 8

EXPERIMENTAL RESULTS OF THE RANDOM SEARCH ALGORITHM

\begin{tabular}{|c|c|c|c|c|c|c|c|c|}
\hline \multirow[t]{2}{*}{ No. of Sites } & \multirow{2}{*}{$\begin{array}{l}\text { No. of } \\
M D O \mathrm{~s}\end{array}$} & \multirow{2}{*}{$\begin{array}{c}\text { No.of } \\
\text { Problems } \\
\end{array}$} & \multirow{2}{*}{$\begin{array}{c}\text { No. of } \\
\text { Opt. Sol. }\end{array}$} & \multirow{2}{*}{$\begin{array}{c}\text { Aver: \% } \\
\text { Deviation }\end{array}$} & \multicolumn{4}{|c|}{ Number of Sol. with deviation in range } \\
\hline & & & & & $(0,5 \%)$ & {$[5,10 \%)$} & {$[10,20 \%)$} & {$[20 \%,-)$} \\
\hline 4 & 4 & 100 & 98 & 11.01 & 1 & $\overline{0}$ & 0 & 1 \\
\hline 4 & 5 & 100 & 90 & 12.72 & 5 & 1 & 1 & 3 \\
\hline 4 & 6 & 100 & 80 & 7.00 & 12 & 3 & 3 & 2 \\
\hline 4 & 7 & 100 & 73 & 4.90 & 19 & 4 & 3 & 1 \\
\hline 4 & 8 & 100 & 70 & 5.10 & 19 & 4 & 6 & 1 \\
\hline 5 & 4 & 100 & 97 & 1.10 & 3 & 0 & 0 & 0 \\
\hline 5 & 5 & 100 & 88 & 4.97 & 9 & 1 & 0 & 2 \\
\hline 5 & 6 & 100 & 77 & 4.24 & 17 & 3 & 2 & 1 \\
\hline 5 & 7 & 100 & 75 & 5.13 & 17 & 5 & 0 & 3 \\
\hline 5 & 8 & 100 & 62 & 5.63 & 26 & 7 & 3 & 2 \\
\hline 6 & 4 & 100 & 92 & 4.89 & 5 & 3 & 0 & 0 \\
\hline 6 & 5 & 100 & 88 & 5.01 & 8 & 3 & 1 & 0 \\
\hline 6 & 6 & 100 & 74 & 3.05 & 21 & 3 & 2 & 0 \\
\hline 6 & 7 & 100 & 68 & 4.08 & 22 & 7 & 3 & 0 \\
\hline 6 & 8 & 100 & 56 & 2.87 & 37 & 5 & 2 & 0 \\
\hline 7 & 4 & 100 & 86 & 1.86 & 13 & 0 & 1 & 0 \\
\hline 7 & 5 & 100 & 84 & 2.63 & 13 & 2 & 1 & 0 \\
\hline 7 & 6 & 100 & 68 & 2.91 & 27 & 3 & 2 & 0 \\
\hline 7 & 7 & 100 & 63 & 3.55 & 27 & 6 & 3 & 1 \\
\hline 7 & 8 & 100 & 62 & 4.49 & 27 & 6 & 4 & 1 \\
\hline 8 & 4 & 100 & 90 & 1.51 & 10 & 0 & 0 & 0 \\
\hline 8 & 5 & 100 & 81 & 2.44 & 16 & 2 & 1 & 0 \\
\hline 8 & 6 & 100 & 68 & 2.34 & 28 & 3 & 1 & 0 \\
\hline 8 & 7 & 100 & 66 & 2.63 & 27 & 7 & 0 & 0 \\
\hline 8 & 8 & 100 & 50 & 3.65 & 40 & 6 & 4 & 0 \\
\hline
\end{tabular}

number of optimal solutions is less but the average deviation is similar. The Hill-Climbing algorithm is a highcomplexity algorithm and is expected to yield better results. On the other hand, the complexity of the Random Search algorithm is low and its performance is satisfactory.

The Random Search algorithm may depend on the values selected for its parameters such as MAXCOUNT,
MAXSTEP, and MARGIN. As mentioned above, these parameters are multiples of the number of MDOs and the number of sites. To study the sensitivity of the algorithm to these parameters and seek the possibility of further improvement in the solution quality, we varied these parameters. The results are plotted in Fig. 14 which indicates the number of optimal solutions 


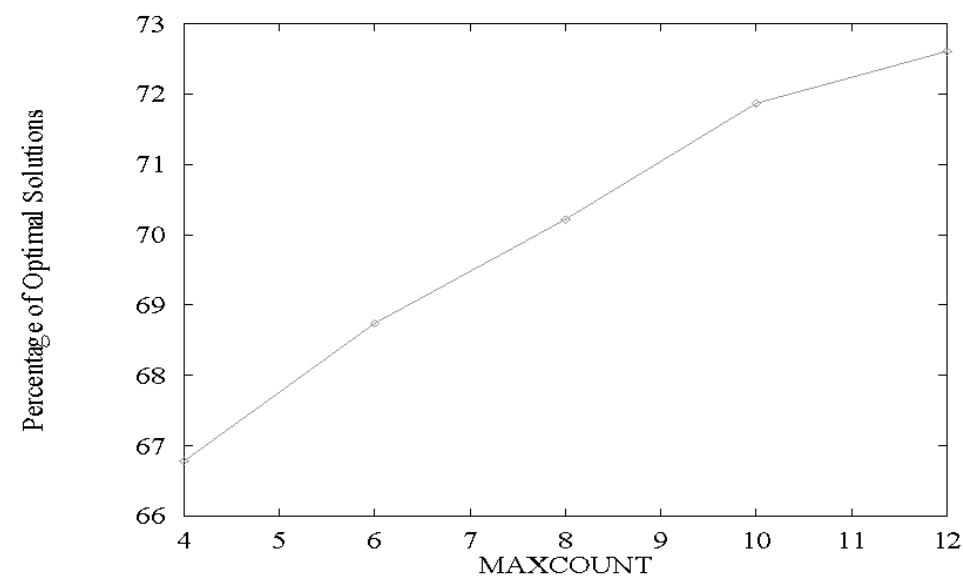

(a)

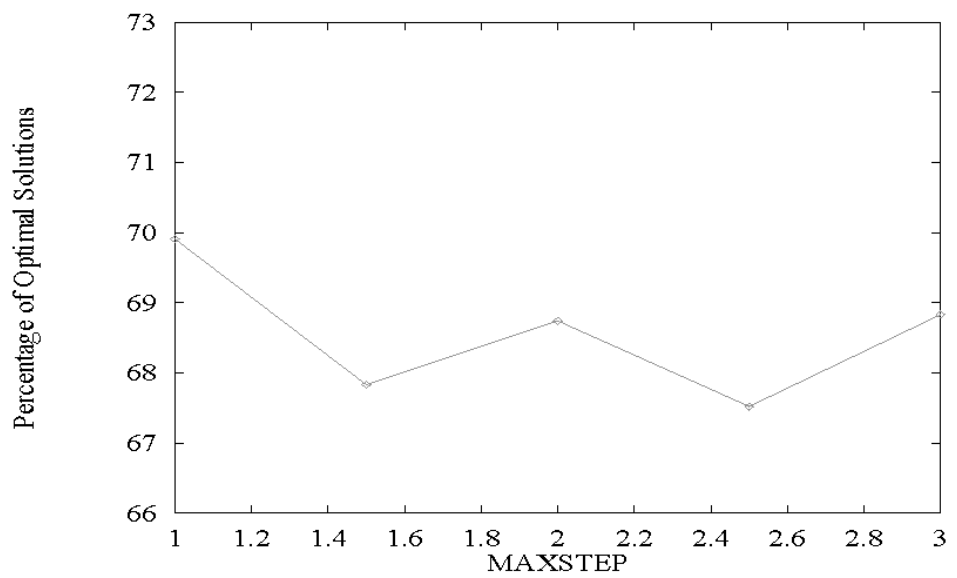

(b)

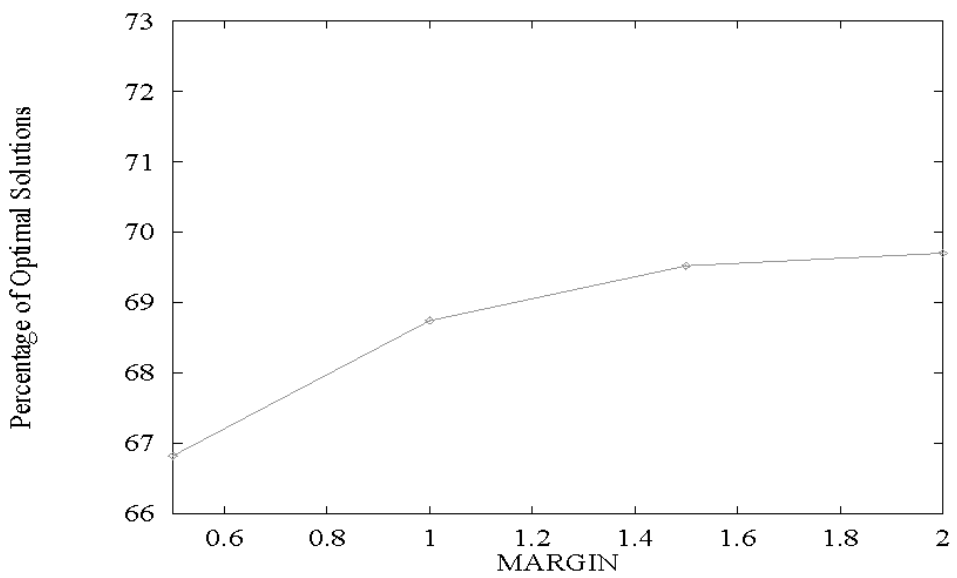

(C)

Fig. 14. Sensitivity of parameters in the random search algorithm: (a) percentage of optimal solutions obtained against MAXCOUNT; (b) percentage of optimal solutions obtained against MAXSTEP; (c) percentage of optimal solutions obtained against MARGIN.

against these numbers. In Fig. 14a, MAXCOUNT is varied from four to 12 times the number of sites multiplied by the number of CMDOs. This figure indicates that MAXCOUNT does have an impact on the performance and with a higher value can yield more optimal solutions. However, the performance saturates beyond a certain range. The parameter MAXSTEP (see Fig. 14b) does not seem to impact the performance and is in fact included in the algorithm to control its searching steps. The MARGIN algorithm (Fig. 14c) does have an impact but, similar to MAXCOUNT, has a certain range of values that are more effective (such as 1.0 to 1.5 ). 
TABLE 9

Average RunNing Times of All Algorithims (IN MSECS)

\begin{tabular}{|c|r||r|r|c|}
\hline No. of Sites & No. of $\boldsymbol{M D O}$ s & Exhaustive Search & Hill Climbing & Random Search \\
\hline \hline 4 & 4 & 7.63 & 38.95 & 27.44 \\
\hline 4 & 5 & 62.99 & 90.42 & 51.37 \\
\hline 4 & 6 & 276.57 & 179.85 & 81.06 \\
\hline 4 & 7 & 1088.42 & 355.07 & 121.39 \\
\hline 4 & 8 & 6457.69 & 1014.08 & 183.12 \\
\hline 5 & 4 & 18.34 & 68.36 & 45.57 \\
\hline 5 & 5 & 134.40 & 153.56 & 81.77 \\
\hline 5 & 6 & 1085.80 & 313.18 & 134.38 \\
\hline 5 & 7 & 8330.39 & 821.18 & 202.27 \\
\hline 5 & 8 & 50224.66 & 2011.78 & 314.17 \\
\hline 6 & 4 & 51.88 & 85.14 & 67.28 \\
\hline 6 & 5 & 529.97 & 236.69 & 120.29 \\
\hline 6 & 6 & 5159.62 & 630.65 & 192.95 \\
\hline 6 & 7 & 38925.23 & 1385.54 & 289.33 \\
\hline 6 & 8 & 273494.96 & 2885.52 & 446.66 \\
\hline 7 & 4 & 176.10 & 166.09 & 97.67 \\
\hline 7 & 5 & 1610.73 & 401.65 & 165.79 \\
\hline 7 & 6 & 22203.47 & 992.97 & 277.62 \\
\hline 7 & 7 & 515439.65 & 2300.59 & 427.97 \\
\hline 7 & 8 & 3387830.28 & 4721.17 & 620.85 \\
\hline 8 & 4 & 3560.16 & 169.28 & 134.06 \\
\hline 8 & 5 & 40439.50 & 567.55 & 217.09 \\
\hline 8 & 6 & 576860.17 & 1520.83 & 355.32 \\
\hline 8 & 7 & 4369.09 & 697.61 \\
\hline 8 & & & 12053.53 & 875.84 \\
\hline & & &
\end{tabular}

\subsection{Comparison of Running Times}

Table 9 contains the average running times of the two algorithms for each experiment. For comparison, the time taken to generate the optimal solutions by using an exhaustive search are also listed. The algorithms were implemented on a SPARC IPX workstation and the timing data was measured in milliseconds. As can be seen from the table, although the Random Search algorithm took much shorter time compared with the exhaustive search and about 1 order of magnitude less time than the Hill-Climbing Approach. Such margins become highly significant when the problem size gets large. Therefore, while the Hill-Climbing algorithm may be preferred for small problem sizes, the Random algorithm would be a better choice for large problem sizes.

From the experimental results presented in the previous section, we observe that there is a trade-off between the execution time and solution quality. The random search algorithm is very cost-effective if fast execution is desired. If the solution quality is the more prominent factor, the Hill-Climbing approach is a viable choice for an off-line allocation.

\section{Related Work}

There is little doubt that the next generation of information processing systems are multimedia in nature and are built on top of a communication network. These resultant systems will be called as distributed multimedia systems $(D M S)$. Multimedia documents are different from the traditional single-media documents in that they have synchronization requirements between different media. One of the problems encountered in DMSs is the lack of specification models for capturing temporal constraints among various objects. Both HyTime [6], [16] and OCPN [13] are developed to solve this problem. Numerous variations in OCPN have been proposed (see [25] for a survey of OCPN and its variants). As the original $O C P N$ model does not incorporate user interactions, an extension called $A O C P N$ is developed in [17] for modeling user interactions such as stop and resume, reverse and terminate.

One limitation of the OCPN model is that there is no explicit way for modeling the navigation path from one document to another. This is because of the restriction of OCPN that each place can only have one outgoing link, but one can browse to many documents from the current document.

In [20], a model called TPN is proposed and can be used to model user interactions that are timed (i.e., can occur only in a predefined period). This model can specify the browsing semantics (when and how). The main problem with this model is that it cannot capture the situation where the user continues to with a document after its first presentation. The time constraint of the model is too strict that when the presentation of a document is ends, that is, one of the transition must be fired (either go to another document or terminate).

The objective of the above two extensions of $O C P N$ is to develop models suitable for interactive multimedia document specifications. However, these models only specify the possible path of document presentation, they do not provide the information about the expected number of times each state (representing $M D O$ or hypermedia document) is needed in a unit time interval. Without this information, the total response time of the DMS cannot be estimated. Another extension of OCPN called XOCPN has been 
proposed which models the object sizes and synchronization at a finer level [26].

Our main contribution is the development of the probabilistic models for navigation and hypermedia presentation. By analyzing these models, we can estimate the total response time of the DMS. We can use the cost function to compare different $M D O$ allocation schemes generated by different allocation algorithms. Our main concern is in finding optimal data allocation scheme for MDOs in different sites of a distributed hypermedia database system such that the total response time of the database system is minimal.

The enforcement of the synchronized multimedia presentation is another important aspect of a DMS. In [14], the general composition (including the spatio and temporal constraints) problem of distributed multimedia objects is discussed and a scheme for mapping the whole composition process to network resources is proposed.

By using the buffers in each site efficiently, the delays of subsequent querying will be reduced significantly if the data requested is still in a buffer. A buffer management scheme for continuous media sharing can be found in [9]. Another component that will have a great effect on the presentation is the network [22]. By partitioning the network bandwidth to channels, we can use the network as efficiently as possible [2]. The original problem considered in [2] is for I/O buffering but the principle can be easily adopted to network channel utilization. If a fault has occurred in the network during a multimedia presentation, the presentation may stop and need to wait for data arrival, which is unacceptable. In [24], a scheme of adaptive presentation management is proposed to be used with slow data arrival. This scheme lowers the presentation quality but ensures that the presentation is carried out smoothly.

\section{Conclusions}

In this paper, we address the problem of response time driven allocation of MDOs for browsing hypermedia documents in distributed environments. This problem addresses both the response time optimization, and adherence to synchronization constraints in the context of data allocation. We develop a probabilistic navigational model for modeling the user behavior while browsing hypermedia documents. This model is used to calculate the expected number of accesses to each hypermedia document from each site. The synchronization constraints for presenting the MDOs of hypermedia documents are modeled by using the OCPN specification. A cost model is developed to calculate the average response time observed by the end users while browsing a set of hypermedia documents for a given allocation of MDOs. This cost model is generalized to take into consideration end-user interaction while accessing MDOs, and limited buffer space constraints at the end-user site. A real-life example is presented to illustrate the utility of the cost model and motivate the need for a good data allocation of MDOs. After that, two MDO data allocation algorithms, one based on Hill-climbing heuristic, and other based on Neighborhood search are proposed.
The two algorithms use extreme approaches:

1) a high complexity extensive incremental strategy, and

2) fast random search.

Results indicate that there is a trade-off between the execution time and solution quality. The neighborhood search algorithm is cost-effective if fast execution is desired. If the solution quality is the more prominent factor, the Hill-Climbing approach is a viable choice for small problem sizes.

There are some other related aspects we have not explored in this paper. Dynamic data allocation is one such aspect. In environment where user behavior change frequently, the allocation scheme must be adapted to maintain the system performance. Another important aspect is data replication. For MDOs that are read only, duplicating them to all the sites needing them can enhance the overall system performance. However, such strategy may not be feasible due to limited storage space. For static allocation scheme, we can employ some variant of the greedy algorithm to give a near optimal data replication scheme. The real challenging situation is to develop dynamic data replication algorithms. There are several methodologies in the literature [27] to attack this problem for reducing data transfer cost, we are currently adapting these approaches to this problem.

\section{REFERENCES}

[1] P.B. Berra, C.Y.R. Chen, A. Ghafoor, C.C. Lin, T.D.C. Little, and D. Shin, "Architecture for Distributed Multimedia Systems," Computer Comm., vol. 13, no. 4, pp. 217-31, May 1990.

[2] S. Chaudhuri, S. Ghandeharizadeh, and C. Shahabi, "Avoiding Retrieval Contention for Composite Multimedia Objects," Proc. VLDB, 21st Int'l Conf. Very Large Data Bases, pp. 287-298, 1995.

[3] R. Erfle, "HyTime as the Multimedia Document Model of Choice," Proc. Int'l Conf. Multimedia Computing and Systems, pp. 445454, 1994.

[4] K.P. Eswaran, "Placement of Records in a File and File Allocation in a Computer Network," Information Processing, pp. 304-307, 1974.

[5] A. Ghafoor, "Multimedia Database Management Systems," ACM Computing Surveys, vol. 27, no. 4, pp. 593-598, Dec. 1995.

[6] C.F. Goldfarb, "Standards-HyTime: A Standard for Structured Hypermedia Interchange," Computer, vol. 24, no. 8, pp. 81-84, Aug. 1991.

[7] E. James, "Media and Hypermedia," Proc. IEE Colloquium on Large Databases in Press and Publishing: The Present and the Future, Digest no. 101 , pp. $1-2,1990$.

[8] D.S. Johnson, C.H. Papadimitriou, and M. Yannakakis, "How Easy is Local Search," J. Computer and System Sciences, vol. 37, no. 1, pp. 79-100, Aug. 1988.

[9] M. Kamath, K. Ramamritham, and D. Towsley, "Continuous Media Sharing in Multimedia Database Systems," Proc. Fourth Int'l Conf. Database Systems for Advanced Applications, pp. 79-86, 1995.

[10] F. Kappe, G. Pani, and F. Schnabel, "The Architecture of a Massively Distributed Hypermedia System," Internet Research, vol. 3, no. 1, pp. 10-24, Spring 1993.

[11] Y.-K. Kwok, I. Ahmad, and J. Gu, "FAST: A Low-Complexity Algorithm for Efficient Scheduling of DAGs on Parallel Processors," Proc. 25th Int'l Conf. Parallel Processing, vol. II, pp. 150-157, Aug. 1996.

[12] Y. Kwok, K. Karlapalem, I. Ahmad, and N.M. Pun, "Design and Evaluation of Data Allocation Algorithms for Distributed Multimedia Database Systems," IEEE J. Selected Areas in Comm., vol. 14, no. 7 , pp. 1,332-1,348, Sept. 1996.

[13] T.D.C. Little, "Synchronization and Storage Models for Multimedia Objects," IEEE J. Selected Areas in Comm., vol. 8, no. 3, pp. 413427, Apr. 1990. 
[14] T.D.C. Little, "Spatio-Temporal Composition of Distributed Multimedia Objects for Value-Added Networks," Computer, vol. 24 , no. 10, pp. 42-50, Oct. 1991

[15] M.A. Marsan, G. Balbo, G. Conte, S. Donatelli, and G. Franceschinis, Modelling with Generalized Stochastic Petri Nets, Wiley, 1995.

[16] S.R. Newcomb, "Multimedia Interchange Using SGML/HyTime," IEEE Multimedia, vol. 2, no. 2, pp. 86-89, Summer 1995.

[17] B. Prabhakaran and S. V. Raghavan, "Synchronization Models for Multimedia Presentation with User Participation," Multimedia Systems, vol. 2, pp. 53-62, 1994.

[18] R. Rada, "Hypertext, Multimedia and Hypermedia," New Review of Hypermedia and Multimedia, Applications, and Research, vol. 1, pp. 1-21, 1995.

[19] G.R. Rao, V. Balasubramanian, and B.A. Suresh, "Integration of Hypertext and Object-Oriented Databases for Information Retrieval," Proc. 1993 IEEE 19th Ann. Northeast Bioeng. Conf., pp. 201204, May 1993.

[20] J. Song, Y.N. Doganata, M.Y. Kim, and A.N. Tantawi, "Modeling Timed User-Interactions in Multimedia Documents," Proc. Int'l Conf. Multimedia Computing and Systems, pp. 407-416, 1996.

[21] P.D. Stotts and R. Furuta, "Petri-Net-Based Hypertext: Document Structure with Browsing Semantics," ACM Trans. Information Systems, vol. 7, no. 1, pp. 3-29, Jan. 1989.

[22] V.S. Subrahmanian, Multimedia Database Systems: Issues and Research Directions, Springer, 1996.

[23] H.M. Taylor and S. Karlin, An Introduction to Stochastic Modeling, Academic Press, 1994.

[24] H. Thimm and W. Klas, " $\delta$-sets for Optimized Reactive Adaptive Playout Management in Distributed Multimedia Database Systems," Proc. 12th Int'l Conf. Data Eng., pp. 584-592, 1996.

[25] S. Vuong, K. Cooper, and M. Ito, "Specification of Synchronization Requirements for Distributed Multimedia Systems," Proc. Int'l Workshop Multimedia Software Development, pp. 110-119, 1996.

[26] M. Woo, N.U. Qazi, and A. Ghafoor, "A Synchronization Framework for Communication of Pre-Orchestrated Multimedia Information," IEEE Network, pp. 52-61, Jan./Feb. 1994.

[27] O. Wolfson, S. Jajodia, and Y. Huang, "An Adaptive Data Replication Algorithm," ACM Trans. Database Systems, vol. 22, no. 2, pp. 55-314, June 1997.

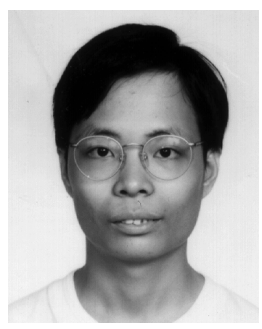

Siu-Kai So received his bachelors degree in computer science from the Hong Kong University of Science and Technology in 1996. Currently, he is now pursuing his masters degree from the same university. His research interests are in the areas of distributed systems, database systems, multimedia applications and algorithm design.

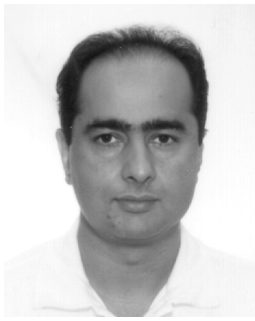

Ishfaq Ahmad received a BSc degree in electrical engineering from the University of Engineering and Technology, Lahore, Pakistan, in 1985; and his MS degree in computer engineering and $\mathrm{PhD}$ degree in computer science, both from Syracuse University, in 1987 and 1992, respectively. He now is an associate professor in the Department of Computer Science at the Hong Kong University of Science and Technology. His research interests are in the areas of parallel programming tools, scheduling, and mapping algorithms for scalable architectures, video technology, and interactive multimedia systems. He has published extensively in the above areas. $\mathrm{He}$ has received numerous research and teaching awards, including the Best Student Paper Award at Supercomputing '90 and Supercomputing '91, and the Teaching Excellence Award of the School of Engineering at the Hong Kong University of Science and Technology. $\mathrm{He}$ has served on the committees of various international conferences, has been a guest editor for two special issues of Concurrency Practice and Experience related to resource management, and is co-guesteditor of a forthcoming special issue of the Journal of Parallel and Distributed Computing on software support for distributed computing. $\mathrm{He}$ serves on the Editorial Board of Cluster Computing. He is a member of the IEEE and the IEEE Computer Society.

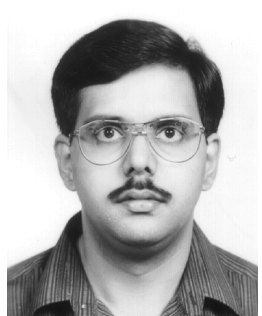

Kamalakar Karlapalem received his MStat degree from the Indian Statistical Institute, Calcutta, India, in 1985; the MTech degree in computer science from the Indian Institute of Technology, Kharagpur, in 1986; and the PhD degree from the Georgia Institute of Technology in December 1992. He has been an assistant professor in the Department of Computer Science at the Hong Kong University of Science and Technology since 1993. He worked for two years as a software engineer at Tata Consultancy Services, India, and the National Informatics Centre, India. He was a student intern at Hewlett-Packard, Cupertino, California, working on conceptualization, design, and implementation of a distributed relational database system. He is interested in the following research areas: cooperative problem solving technology, data warehousing and data mining, distributed database design/redesign, multimedia data allocation, object-oriented class partitioning and workflow systems, and electronic commerce security. He is a member of the IEEE, the IEEE Computer Society, and ACM. 